\title{
Non-Paradoxes of Set Theory and the Diagonal Method
}

\author{
Mark Owens
}

Public Reference Version

Digital Object Identifier (DOI): 10.31219/osf.io/my3k5

Date: 2023-04-22Z

\begin{abstract}
The requirement for axiomatic set theory rests on the belief that the unrestricted comprehension of sets can result in sets that are paradoxical and therefore unrestricted comprehension needs to be tamed. Some key paradoxes of Burali-Forti, Russell and Cantor are examined and shown not to be paradoxes. Additionally, a common technique known as the diagonal method is shown to never construct a contradictory sequence. Then it is shown that any infinite set can be placed in a bijection with its power set by explicitly constructing a bijection and that the real numbers and natural numbers have the same cardinality. Finally, semantic diagonal techniques are considered with particular reference to Gödel's incompleteness theorem and the halting problem.
\end{abstract}




\section{Table of Contents}

1 Introduction

2 The Burali-Forti Paradox

3 Russell's Paradox

4 The Diagonal Method

5 Cantors's Paradox and Cantor's Theorem

6 Semantic Diagonalization

7 References 


\section{Introduction}

There should be no paradoxes in mathematics. Mathematics is a landscape of tautologies consistent with the foundational law of non-contradiction. Being able to prove the truth of any mathematical statement relies on this property. A paradox is a violation of the law of non-contradiction and as such cannot be permitted. Valid mathematical objects and operations are only those consistent with the law of non-contradiction.

Any valid mathematical operation on any valid mathematical object cannot produce a paradox. Arriving at a contradiction is evidence that a mistake has been made, but provides no clue as to where the mistake has been made. Consequently, a proof by contradiction carries the risk of the wrong area being identified as to where the mistake was made, due to containing a selection bias towards the starting assumptions. A valid proof by contradiction implies there should be a proof for the opposite case. Such a proof would provide independent corroboration, although it may be more difficult to construct. The longer a proof by contradiction stands with no proof of the opposite case, the more likely it is to be worthwhile spending effort to explore whether it is correct. So long as there is no corroborating proof for the opposite case, there is always the chance that there is no such proof because the opposite case is not true. Cantor's two proofs of the uncountability of the reals [Cantor,1874], [Cantor,1891] and the proof of Cantor's power set theorem [Hessenberg,1906] are all proofs by contradiction. They are reviewed here and show not to be correct. All of the proofs are related to the diagonal method and this is shown to be the source of the error.

Even when valid, when no mistakes in the internal connections of a chain of reasoning are made, a contradiction at the end of the chain merely provides information on the logical status of a starting proposition; that it must be the opposite of what was initially assumed. It provides no information on why that is the case. In order for the reason why not to be circular there must be a logically prior and independent reason for why that is the case. The why cannot be the contradiction, since that would involve a circular chain of reasoning. To say, for example, that a proposition is false because if it were true it would result in a contradiction is the same as saying that it is false because it cannot be true which is the same as saying that it is false because it is false. In a fully deductive logic system, the logical status of each downstream node is fully determined by upstream nodes and it is the downstream path to any proposition that provides the reason why. The logical status of a proposition can be inferred from downstream consequences but it cannot be deduced. Logical deduction is the act of determining the logical status of a proposition solely from prior information. Any path that leads to a contradiction implies an error lies somewhere upstream. A contradiction is the endpoint of a path that should never have been taken.

When paradoxes began to emerge in set theory, the "mistake" that was identified was in the concept of the set itself, despite the fact that a set, which is nothing more than a collection of objects, has an entirely non-contradictory definition. The standard approach has been to restrict what type of collection can be a set, which results in a collection hierarchy. This approach can be criticized as creating contradictions of its own [Weir,1998]. The essential point is that there is no independent frame of reference from which to classify the hierarchies 
and so the classification is ultimately circular in the manner described in the preceding paragraph. Some examples are considered here.

Instead of correctly identifying the source of set paradoxes, axiomatic set theory makes use of the foreknowledge of apparent downstream contradictions in a logical network to engineer a set of rules that pre-emptively block off certain downstream paths, but does so in a way that cannot be justified on the basis of any logically prior information and in so doing abandons any claims of being fully deductive. It prioritizes safety over logical deduction. Worse, if any axiom, anywhere, in any subject, is not provably true or provably a free choice, so that it is merely a useful component in a classification system, it may actually be false. In a truly deductive system, all axioms must either be a free choice or derivable solely from logically prior information, with the ultimate prior information being the primary laws of logic. This is the essence of the idea underlying the work of Gottlob Frege to root all of mathematics in logic. Frege's work was undermined at the time by Russell's paradox, which is reviewed here, which suggested such an approach would lead to contradictions. Gödel's incompleteness theorem is also a challenge to such an approach as it suggests the existence of correct statements that are not provable and so Gödel's incompleteness theorem is also reviewed here. The halting problem is closely related to Gödel's incompleteness theorem and so that is also reviewed.

Only naive set theory satisfies the condition of being fully deductive, by being nothing more than the application of boolean algebra to the free choice of a collection. A set is the only collection type amenable to boolean algebra. Consequently, a correct application of naive set theory cannot possibly produce a paradox. If a paradox is obtained, the error must lie elsewhere. A selection of some of the key paradoxes are reviewed here and shown not to be the result of the use of sets and specifically not the result of unrestricted comprehension.

Ultimately, it is not set theory that is problematic, but the current understanding of the nature of infinity, leading to a confusion over issues of being countable and well-founded. In the standard approach there are countable, well-founded infinities, which is an oxymoron, and uncountable non-well-founded infinities. Here it is shown that all infinities are countable in the sense that all infinite sets can be placed in a bijection with the natural numbers, but only well-founded subsets can be physically counted. Alternatively, all infinite sets are not countable in the sense that they are not well-founded. 


\section{The Burali-Forti Paradox}

Consider the set of natural numbers. A concrete representation of this set is given by the Von Neumann ordinals.

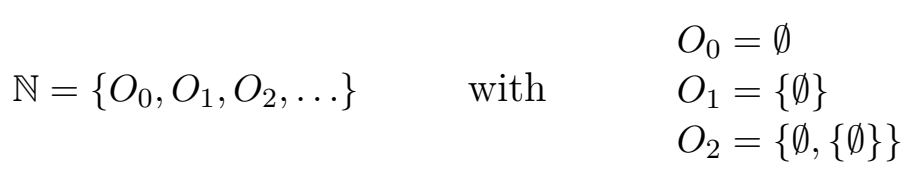

The members of the set can be defined recursively, as

$$
O_{n+1}=O_{n} \cup\left\{O_{n}\right\} \quad \text { with } \quad O_{0}=\emptyset
$$

as can the construction of the whole set. If $\mathbb{N}_{n}$ is the set of ordinals up to $n$, then

$$
\mathbb{N}_{n+1}=\mathbb{N}_{n} \cup\left\{\mathbb{N}_{n}\right\} \quad \text { with } \quad \mathbb{N}_{0}=\{\emptyset\}
$$

The function on set $S$ given as

$$
S^{+}(S)=S \cup\{S\}
$$

is called the successor function and it generates the next highest ordinal. The construction has the feature that

$$
O_{n+1}=\mathbb{N}_{n}
$$

namely that a set of ordinals up to some finite ordinal is equal, in construction, to what will be the next highest ordinal in the sequence. The successor function can be undone by removing the largest member. The inverse of the successor function can be given by

$$
S^{-}(S)=X \subset S: X=S \backslash\{X\}
$$

The complete set of ordinals can be described in predicate form by defining the predicate $O_{v n}()$ to be true for any of the $O_{n}$ Von Neumann ordinals

$$
\mathbb{N}=\left\{S \mid O_{v n}(S)\right\}
$$

This is the set of all ordinal sets. A problem arises when considering if $\mathbb{N}$ is an ordinal. If it is not an ordinal, then there is no problem, but the standard approach considers that is an ordinal due to Equation 2.5 apparently implying that it is the next ordinal after $\mathbb{N}$. If it is an ordinal, then the set contains itself, which means it must represent an ordinal that is less than itself, because ordinals are specifically constructed so that set containment provides the order relationship. But if it doesn't contain itself, then there is a higher ordinal that is not in $\mathbb{N}$. It appears as if the set of all natural numbers should define an even higher natural number. There cannot be a set of all natural numbers if the set itself describes a higher natural number. The set of natural numbers appears to be a paradoxical set. 
The standard approach is to define that $\mathbb{N}$ is an ordinal and so $O_{v n}(\mathbb{N})$ is true, but it is not a natural number. It is the smallest ordinal greater than any natural number, which is designated by the symbol $\omega$. Equation 2.7 then needs to be modified with an additional, ultimately circular, qualification, such as $S<\mathbb{N}$, to ensure that $\mathbb{N}$ only contains natural number ordinals. Note that although the "set of well-founded ordinals" has a none circular description it excludes $S^{-}(\mathbb{N}), S^{-}\left(S^{-}(\mathbb{N})\right)$ and so on. The property of being well-founded is equivalent to there being no infinite descending chains back to the minimal member and so is associated with a count back. It is not possible to reach the end of an infinite sequence in order to start a count back. A count back can only be achieved in a dynamical fashion by counting back on a finite sequence and then letting the sequence tend to infinity, while maintaining the count back on the sequence. The resulting sequence will not be well-founded since there will always be at least one member, that is the member counted back from, that is separated from the minimal member by an infinite sequence.

Nevertheless, the standard approach is successful at describing sequences containing infinite lists. Writing $\omega$ as $\{N\}$ with $N$ representing every member in the set of natural numbers $(\omega=\mathbb{N}=\{N\})$ shows how the successor function continues the ordinal count.

$$
\omega+1=S^{+}(\omega)=\{N\} \cup\{\omega\}=\{N, \omega\}
$$

The ordinal sequence continues from $\omega$ with repeated actions of the successor function. This enables a measure of position in an ordered list for lists composed of infinite sublists, such as

$$
\begin{array}{lll}
\{0,1,2,3,4,5, \ldots, 1\} & \text { with order } & \omega+1 \\
\{0,2,4, \ldots, 1,3,5, \ldots\} & \text { with order } & \omega+\omega
\end{array}
$$

But now a failure to properly deal with the status of $\mathbb{N}$ leads to an unavoidable paradox. This is the Burali-Forti paradox. The set of all ordinals, now designated by $\Omega$ to distinguish it from $\mathbb{N}$, is an ordinal and so must contain itself, but that means it must represent an ordinal that is less than itself, because ordinals are specifically constructed so that set containment provides the order relationship. But if it is not contained in itself it becomes a paradox with respect to the property of being an ordinal. If the set of all ordinals is an ordinal and it is not contained in itself, then there is a higher ordinal that is not in the set of all ordinals. Note that neither case would apply if the set of all ordinals is not an ordinal.

This is the exact same problem that existed with the set of natural numbers, except that now there should be no get out of an appeal to a higher count type, for the set of all ordinals describes every possible ordered sequence. However, the standard resolution is to declare that the set of all ordinals is not a set, but a proper class and in so doing invents a new type. This is a form of avoidance by definition and is ultimately circular since the sole justification is to avoid a contradiction. $\Omega$ is not in the set of all ordinals because it is not a set, it is a proper class. Why is it a proper class? Because otherwise it would be in the set of all ordinals. This results in the self fulfilling statement: $\Omega$ is not in the set of all ordinals because it is not in the set of all ordinals. But it is still an ordinal. In the standard approach the count continues up through collections that are deemed not to be sets with the paradox being repeatedly encountered for each new collection type. 
The paradox automatically resolves itself at the first instance it occurs, which is at $\mathbb{N}$, if a set of ordinals is not itself an ordinal. Equation 2.5 does not define a sequence of ordinals to be an ordinal, but to be the next ordinal in the sequence. A sequence of ordinals will not be an ordinal if there is no next ordinal in the sequence. The essential aspect of this approach is that an infinite sequence is treated as a finite sequence that is forever growing in length and infinity is not an end state that can be meaningfully operated on. That is, one must apply all operations to a finite sequence before taking the limit. Crucially, infinity is treated here as a dynamical entity throughout and not a static value that can be operated on. $\mathbb{N}$ is given by

$$
\mathbb{N}=\lim _{n \rightarrow \infty}\left(S^{+}\right)^{n}(\emptyset)=\{\emptyset,\{\emptyset\},\{\emptyset,\{\emptyset\}\}, \ldots\}
$$

Applying the successor function to $\mathbb{N}$ gives

$$
S^{+}(\mathbb{N})=\lim _{n \rightarrow \infty}\left(S^{+}\right)^{n}(\{\emptyset\})=\{\emptyset,\{\emptyset\},\{\emptyset,\{\emptyset\}\},\{\emptyset,\{\emptyset\},\{\emptyset,\{\emptyset\}\}\}, \ldots\}
$$

The $S^{+}(\mathbb{N})$ sequence is always further ahead of the $\mathbb{N}$ sequence by one count. They are never the same sequence, but they are both tending towards the same limit and ultimate count and so the count has an upper bound given by

$$
S^{+}(\mathbb{N}) \rightarrow \mathbb{N}
$$

Thus, it is not possible to count past the end of an infinite sequence. Note that $S^{-}(\mathbb{N})$ is also tending to $\mathbb{N}$ and so neither is it possible to count back from the end of an infinite sequence to a finite location in a finite number of steps.

While any finite set of natural numbers defines what the next natural number is going to be, an infinite set does not. An infinite set has no termination for such a succession to the next natural to be meaningful in the same way that Hilbert's infinite hotel has an infinite capacity for more guests and it makes no sense to ask what is the next highest room above the top of the hotel. There is no top.

For $\mathbb{N}$, there is no next ordinal and so $O_{v n}(\mathbb{N})$ is false and $\mathbb{N}$ is not an ordinal. Even for a finite sequence of ordinals, it will not be an ordinal unless it is part of a counting sequence. Equation 2.5 does not say that $\mathbb{N}_{n}$ is an ordinal. It says that the next ordinal in a finite sequence of ordinals will be a copy of the current sequence. For any partial finite construction of a universe, the longest ordinal sequence for that universe will not be an ordinal within that universe. For example, if the longest sequence is $\mathbb{N}_{n}$, the ordinal count ends at $O_{n}$. The ordinal count for $O_{n+1}$ does not exist within the sequence or anywhere else in the universe. Thus $O_{v n}(S)$ is not true solely based on the structure of $S$ and is conditional on $S$ appearing somewhere within a counting sequence. This must be the case, otherwise there would be an inconsistency between a longest sequence of any length represented by $O_{v n}\left(\mathbb{N}_{n}\right)$ and a longest sequence of infinite length represented by $O_{v n}(\mathbb{N})$, which is false. Thus, $O_{v n}\left(O_{n}\right)$ is false unless $\mathbb{N}_{n}$ exists and $O_{v n}\left(\mathbb{N}_{n}\right)$ is false unless $\mathbb{N}_{n+1}$ exists. Given that $\mathbb{N}$ can never be surpassed, then this implies and is implied by $O_{v n}(\mathbb{N})$ being false and the top of a Von Neumann universe is the first level that contains $\mathbb{N}$, which will never be reached. 
The standard approach makes no distinction between an ordinal and a sequence of ordinals and so an ordinal can have a limit. The various definitions of a limit ordinal are just different ways to describe an infinite sequence relative to some fixed chosen member and do not apply when selecting the last member of the sequence as $S^{-}(\mathbb{N})$ in a count back since that will not be a fixed member. An explicit example is given at the end of Section 5. In this approach, a set is only an ordinal if it is a member of a counting sequence and it is the sequence that has the limit. A limit ordinal-sequence is defined here as one that is not also an ordinal. For any construction of a universe, at any stage of construction, the longest set of continuous ordinals is a limit ordinal-sequence for the current stage of construction.

Consider how sequences containing infinite lists can be described if there is no count after $\mathbb{N}$. It is not possible count past the end of a sequence that never ends. However, as the limit of $\mathbb{N}$ is approached, it contains members that are also tending to $\mathbb{N}$, which also contains members that are tending to $\mathbb{N}$ and so on. The sets $\mathbb{N}$ and $\Omega$ are just different representations of the same set, which is a matter of choice. The difference in representation is shown for some finite sequence of natural numbers in Equation 2.13.

$$
\begin{aligned}
\mathbb{N}_{9} & =\{0,1, \ldots, 9\} \\
& =\{0,1, \ldots, 8,\{0,1, \ldots, 8\}\} \\
& =\{0,1, \ldots, 7,\{0,1, \ldots, 7\},\{0,1, \ldots, 7,\{0,1, \ldots, 7\}\}\}
\end{aligned}
$$

For a finite sequence, each expansion of a member reduces the count by one. In the infinite limit, all the sequences in Equation 2.13 are tending to infinity and the last line of Equation 2.13 is tending to a representation of the ordinal $\omega+2$. In this description, $\omega$ is a floating marker of an infinite sequence in which the longest sequence is always $\mathbb{N}$. That is, $\omega_{l}=\omega_{s}+2$, where $\omega_{s}$ is the shorter sequence and $\omega_{l}$ is the longer sequence, which is always $\mathbb{N}$. Essentially, one is free to choose a subset of $\mathbb{N}$ that is also tending to infinity, label that as $\omega$ and relabel subsequent sets consistently via Equation 2.8. This is what Equation 2.13 enables. Such an $\omega$ is a proper subset of $\mathbb{N}$ that is also in the process of tending to $\mathbb{N}$, but is always further behind. Thus, $\omega+1$ is not the count after $\mathbb{N}$, but a representation of the relative lengths of two sequences tending to infinity, from the perspective of the shorter sequence, in which one sequence is always 1 ahead of the other. One may choose the longer sequence as a reference sequence to obtain $\omega-1$. This description has commutative arithmetic just the same as with finite numbers. That is, $\omega+1=1+\omega$. There is a limit to this process. One is only free to subdivide an infinite sequence into infinite subsequences up to a relative ordinal less than $\omega_{s}{ }^{\omega_{l}}$, since $n^{1 / n} \rightarrow 1$ as $n \rightarrow \infty$. That is, for the relationship $\omega_{l}=\omega_{s}{ }^{n} \Leftrightarrow \omega_{s}=\omega_{l}^{1 / n}$, $n$ must be very much less than $\omega_{l}$. However, this imposes no restrictions on an $\omega_{l}$ that is only a function of $\omega_{s}$.

Thus, the richness of the structure of infinite ordinals is fully contained within $\mathbb{N}$ and so $\mathbb{N}$ is not well-founded. It has a count that can never be surpassed and so $\mathbb{N}$ is not an ordinal and the Burali-Forti paradox is not a paradox. The issue will be re-examined from the perspective of the identity map of $\mathbb{N}$ at the end of Section 5 . 


\section{Russell's Paradox}

Russell's paradox is an argument against unrestricted comprehension. With unrestricted comprehension, a set $\mathrm{X}$ can be described in predicate form as

$$
X=\left\{S \mid \Phi_{X}(S)\right\}
$$

Bertrand Russell observed that there was a problem with this definition. He proposed to consider the set of all sets that do not contain themselves. Most sets do not contain themselves. For most sets, their definition is either too specific or the property of their members is not heritable. For example, the set of integers from 3 to 6 is too specific a definition. The set of square objects is not a square. The property of squareness is not heritable. But it is conceivable for some sets to appear to qualify as being members of themselves. For example, a set of all wooden objects is a wooden object. The Russell set ignores all such sets and considers only those that do not contain themselves.

$$
R=\{S \mid S \notin S\}
$$

This results in a paradox induced by a self reference if $\mathrm{S}$ is allowed to be $\mathrm{R}$. If $\mathrm{R}$ does not contain itself then it belongs in $\mathrm{R}$ and so must contain itself and if $\mathrm{R}$ does contain itself then it is in $\mathrm{R}$ but $\mathrm{R}$ can only contain sets that do not contain themselves.

Typical approaches to deal with the problem involve either preventing $\mathrm{S}$ from being set to $\mathrm{R}$ through some form of restricted comprehension rules or by defining a collection type that is not a set, such as a proper class, and declaring that $\mathrm{R}$ must be of that type. Both are forms of avoidance by definition and are ultimately circular:

- Assigning $R$ to be a proper class creates a circularity. $R$ is not in the set of all non-selfcontaining sets because it is not a set, it is a proper class. Why is it a proper class? Because otherwise it might be in the set of all non-self-containing sets. This results in the self fulfilling statement: $R$ is not in the set of all non-self-containing sets because it is not in the set of all non-self-containing sets.

- Restricting $S$ from being set to $R$ can only be done through the use of additional axioms that have no logically prior basis. One axiom, at least, must be forwards looking. Their ultimate justification is that they avoid a paradox, which makes their use no less circular than the use of proper classes. $S$ cannot be set to $R$ because an axiom prevents it. Why does an axiom prevent it? Because otherwise $S$ could be set to $R$.

The set membership operator implies a set container which raises the rank over the rank of those that are contained. If a set $\mathrm{B}$ is in a set $\mathrm{A}$, then $\mathrm{B}$ is a member of $\mathrm{A}$. This means that, by definition, $\operatorname{Rank}(A)>\operatorname{Rank}(B)$. For a set $\mathrm{S}$ to be a member of itself it would require $\operatorname{Rank}(S)>\operatorname{Rank}(S)$ which can never be true. There can be no equality for sets with an infinite containment hierarchy since the rank of a set is defined to be the least ordinal number greater than the rank of any member of the set. The rank of a container is always greater than the rank of anything it contains. This is related to the Burali-Forti paradox, since the rank of an ordinal set is the same as its ordinal value. Except that now it applies to 
every set that might contain itself since every set has a rank. Thus, a set can never contain itself. So Russell's set reduces to the set of all sets and the paradox disappears. This gives rise to a new theorem:

\section{Theorem 3.1: Self Comprehension Set Theorem}

- The comprehension for any set with respect to itself is always false.

- For any set $\mathrm{X}$ with unrestricted comprehension $\forall X=\left\{S \mid \Phi_{X}(S)\right\}: \Phi_{X}(X)$ is false

A question arises as to how to consistently describe, in this framework, those sets that definitely appear to qualify as being members of themselves. Take, for example, the set of all wooden objects. The set has the property that any combination of members of the partial completion of the set qualifies for membership in the set. Such sets are highly pathological, trapped in an infinite loop of forever adding new sets composed from the existing membership. Consistency with Theorem 3.1 implies that there should be a separation between set containment and the property that a set has. The "of" in "set of" implies objects that can be members of the set in question and thus contained by such a set. If $\Gamma_{X}(S)$ is the predicate that $\mathrm{S}$ could be contained somewhere in $\mathrm{X}$ due to having a lower rank and $P_{x}(S)$ is the predicate for the property that builds $\mathrm{X}$, then

$$
\Phi_{X}(S)=\Gamma_{X}(S) \wedge P_{x}(S)
$$

The predicate $\Phi_{X}(X)$ is always false because $\Gamma_{X}(X)$ is always false. $P_{x}(X)$ can take any value. In terms of propositional logic the situation is similar to the statement "The king of France is bald" which does not cover all possibilities and so violates the law of excluded middle. All possibilities are covered with the conjunction "There is a king of France and he is bald". Thus, even though the set of all wooden objects is a wooden object, it does not contain itself. It is trapped in an infinite loop of forever trying to contain itself, but never succeeding. The set is actually the set of all wooden objects containable in itself and it is not containable in itself so the set is consistent with respect to both containment and its property. This raises the question of whether the set is containable in any set.

To explore the issue further it is instructive to introduce some definitions in order to deliberately attempt to re-introduce Russell's paradox, in this framework. A normal set is defined to be a set that does not inherit the property of its members and an abnormal set is one which does. If $P_{N}(S)$ is the predicate that set $\mathrm{S}$ is normal, then the closest possible object to the original definition of the Russell is the set of all normal sets (call this $R^{\prime}$ ) which is given by:

$$
R^{\prime}=\left\{S \mid \Gamma_{R^{\prime}}(S) \wedge P_{N}(S)\right\}
$$

There is no longer any paradox with respect to self containment since $\Gamma_{R^{\prime}}\left(R^{\prime}\right)$ is false. But the paradox has re-emerged with respect to the property of being normal. If the set of all normal sets is normal then it has the same property of its members and so should be abnormal. If the set of all normal sets is abnormal then it does not have the same property 
of its members and so should be normal. This does not appear to be an improvement on the original version of the paradox since it has merely been shifted from the containment predicate $\Gamma_{R^{\prime}}()$ to the property predicate $P_{N}()$. However, while containment is an essential property of sets, the use of logical predicates is not. Thus, the paradox is not the result of the use of sets, but the result of the use of logical propositions.

It is easy to construct paradoxical logical propositions. One merely needs to refer an object to itself via some process of negation. The classic example is the statement "this sentence is false". The logical proposition equivalent of Russell's paradox that was publicised by Russell is the barber paradox: "The barber shaves all those and only those who do not shave themselves". So if the barber shaves themself then they must not shave themself and visa versa. This can be deconstructed into the conjunction of three propositions "(1) There is a barber and (2) the barber shaves all those others who do not shave themselves and (3) if the barber shaves themself then the barber does not shave themself". Proposition 3 is a clear violation of the law of non-contradiction. If proposition 3 is retained then proposition 1 must be false.

Given that a proof by contradiction embraces a contradiction instead of trying to resolve it, such a proof must take special care that it is not set up in such a way as to guarantee the contradiction. If the proof is set up in such a way as to make an object equal to the negation of itself, then a contradiction is guaranteed. If the process involved in the setup can be physically carried out then there must be something in the way the process works that negates the contradiction even if it is not obvious from the outset.

The property of being normal is defined in terms of whether a set inherits the property of its members. The self reference comes from making itself the property being considered. The negation comes from considering those that do not inherit. The negation is significant, for without the negation there is no paradox. That is, the set of all abnormal sets is not paradoxical. If a set of abnormal sets is abnormal then it inherits the property of its members. If a set of abnormal sets is normal then it does not inherit the property of its members. It appears one is only free to consider the heritable property of sets if the property in question is not the property of inheritance. Is this a sufficient resolution of the paradox? It is not, for one is certainly free to gather together a collection of sets that do not inherit from their members, with $\mathbb{N}$ being one such example. It should be possible to consistently investigate any property this collection may have. Specifically, it must be possible to obtain a value for $P_{N}\left(R^{\prime}\right)$. Since the process can be physically carried out there must be something about the way it works that is not yet understood.

Putting the issue aside for the time being, in this framework, the universe is the set of all sets containable in itself.

$$
U=\left\{S \mid \Gamma_{U}(S)\right\}
$$

An interesting question arises as to whether this is any different from an enumeration over all sets. Can there be any sets that are not containable in $U$ ? One can investigate the relationship between a set's property and set containment by partitioning $U$ with respect to 
some property p:

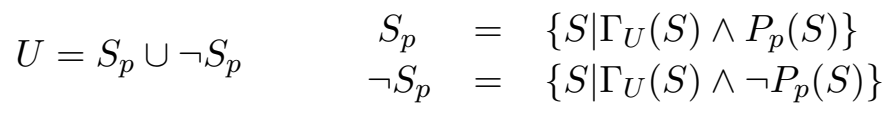

Here "partition" includes the possibility of a null partition into the empty set and the original set. The partition sets, $S_{p}$ and $\neg S_{p}$, may or may not exist as sets that can be contained and may only exist as a partition of the universe. That is, $\Gamma_{U}()$ will be false for a partition set if its members only exist in a partition of the universe. The partition sets cannot exist inside themselves as no set can contain itself. They can only be contained, if at all, in their complement. Both partition sets cannot simultaneously be contained as that would also result in them containing themselves within their own hierarchy. So at most $\Gamma_{U}()$ can only be true for one of the partition sets. The value of $\Gamma_{U}()$ for $S_{p}$ and $\neg S_{p}$ can be inferred from their properties. Writing the complement property as $P_{c}(S)=\neg P_{p}(S)$ and the complement set as $S_{c}=\neg S_{p}$, then the comprehension of the partition sets is given by

$$
\begin{aligned}
& \Phi_{S_{p}}(S)=\Gamma_{U}(S) \wedge P_{p}(S) \\
& \Phi_{S_{c}}(S)=\Gamma_{U}(S) \wedge P_{c}(S)
\end{aligned}
$$

Applying Theorem 3.1 to the partition sets gives

$$
\begin{aligned}
& \Gamma_{U}\left(S_{p}\right) \wedge P_{p}\left(S_{p}\right)=\text { false } \\
& \Gamma_{U}\left(S_{c}\right) \wedge P_{c}\left(S_{c}\right)=\text { false }
\end{aligned}
$$

Whenever $P_{p}\left(S_{p}\right)$ is true or $P_{c}\left(S_{c}\right)$ is true, then that partition inherits the property of its members and its corresponding $\Gamma_{U}()$ must be false. In general, there are a total of 16 possible configurations. 8 can be eliminated, since they are inconsistent with the above required properties. The remaining 8 configurations can be classified into 3 types.

Four configurations describe a solution that is symmetric with respect to the transformation $P_{p^{\prime}}()=P_{c}()$. It is the only symmetric solution in which some sets are containable. Any set that does not inherit the property of its members is containable, but only if its complement set does inherit.

\begin{tabular}{|cc|cc|}
\hline$P_{p}\left(S_{p}\right)$ & $P_{c}\left(S_{c}\right)$ & $\Gamma_{U}\left(S_{p}\right)$ & $\Gamma_{U}\left(S_{c}\right)$ \\
\hline $\mathrm{F}$ & $\mathrm{F}$ & $\mathrm{F}$ & $\mathrm{F}$ \\
$\mathrm{F}$ & $\mathrm{T}$ & $\mathrm{T}$ & $\mathrm{F}$ \\
$\mathrm{T}$ & $\mathrm{F}$ & $\mathrm{F}$ & $\mathrm{T}$ \\
$\mathrm{T}$ & $\mathrm{T}$ & $\mathrm{F}$ & $\mathrm{F}$ \\
\hline
\end{tabular}

$\Gamma_{U}(S)=\neg\left(P_{s}(S) \vee P_{s}(\neg S)\right)$

where $s$ is the property that

constructs $S$

An example of both partitions not inheriting is given by the property of containing the empty set as a member. Another example is the property of having an even rank. The case of only one partition not inheriting will be shown in Section 5 to apply to every member of $U$. An example of both partitions inheriting is the property of being built from a set that is not the empty set. The built-from set may itself be built from the empty set and will be in a Von Neumann universe, but the built-from test stops peeling back layers when the built-from set is reached and every reached set must be the built-from set for the property to be true. 
Two configurations describe an antisymmetric solution in which both inheritance properties are always false, but only result in one non-containable set. The containability depends on the containability of the other set.

\begin{tabular}{|cc|cc|}
\hline$P_{p}\left(S_{p}\right)$ & $P_{c}\left(S_{c}\right)$ & $\Gamma_{U}\left(S_{p}\right)$ & $\Gamma_{U}\left(S_{c}\right)$ \\
\hline $\mathrm{F}$ & $\mathrm{F}$ & $\mathrm{F}$ & $\mathrm{T}$ \\
$\mathrm{F}$ & $\mathrm{F}$ & $\mathrm{T}$ & $\mathrm{F}$ \\
\hline
\end{tabular}

$$
\Gamma_{U}(S)=\neg \Gamma_{U}(\neg S)
$$

The self-referential nature of the solution is the result of a self-referential inheritance property, of which there is only one. The property of inheriting. This will be shown to be the case for the property of being normal/abnormal.

The remaining two configurations replace two of the previous symmetric configurations to describe a uniform solution in which both partitions are not containable for any possible configuration of the inheritance properties of the partitions.

\begin{tabular}{|cc|cc|}
\hline$P_{p}\left(S_{p}\right)$ & $P_{c}\left(S_{c}\right)$ & $\Gamma_{U}\left(S_{p}\right)$ & $\Gamma_{U}\left(S_{c}\right)$ \\
\hline $\mathrm{F}$ & $\mathrm{F}$ & $\mathrm{F}$ & $\mathrm{F}$ \\
$\mathrm{F}$ & $\mathrm{T}$ & $\mathrm{F}$ & $\mathrm{F}$ \\
$\mathrm{T}$ & $\mathrm{F}$ & $\mathrm{F}$ & $\mathrm{F}$ \\
$\mathrm{T}$ & $\mathrm{T}$ & $\mathrm{F}$ & $\mathrm{F}$ \\
\hline
\end{tabular}

$$
\Gamma_{U}(S)=\text { false }
$$

It describes the non-static case where the inheritance property of a partition is forever changing. This is the situation for the property of having an even number of members.

A collection that is too large to be contained in any set is the essential property of what is commonly called a proper class. Crucially, though, a proper class is still a set. It is just a special kind of set that cannot be contained in any set. The above provides for a non-circular way to define a proper class, through the following theorem:

\section{Theorem 3.2: Proper Class Theorem}

- A set that has a non-static inheritance property is not containable in any set

- A set that has a static inheritance property, that is not the property of inheriting, is not containable in any set if it:

- either inherits the property of its members

- or neither it nor its complement inherit the property of its members.

For any such set $\mathrm{X}$ defined by property predicate $P_{x}$

$$
\forall X=\left\{S \mid \Phi_{X}(S)=\Gamma_{U}(S) \wedge P_{x}(S)\right\}: \Gamma_{U}(X)=\neg\left(P_{x}(X) \vee P_{x}(\neg X)\right)
$$

Following on from Theorem 3.2, a proper class can be defined as:

\section{Definition 3.1: Proper Class}

- A proper class is a set that is not containable in any set. 
The set of all wooden objects is a wooden object and so is a proper class, as is its complement. $\mathbb{N}$ is not a proper class since it does not inherit the property of its members and its complement set does. $\mathbb{N}$ exists in its complement set as itself, since $\mathbb{N}$ is not a natural number. This is only consistent with an upper count limit of $\mathbb{N}$ if a set of ordinals is not itself an ordinal unless it is part of a counting sequence somewhere, so that a Von Neumann universe always contains as members, at any stage of construction, some number of separate ordinal sets associated with the $\mathbb{N}$ partition and a set containing those same ordinals associated with the $\neg \mathbb{N}$ partition. In terms of Theorem 3.2, for the $\mathbb{N}$ partition of $U$ :

$$
\begin{aligned}
& \mathbb{N}=\left\{S \mid \Gamma_{U}(S) \wedge O_{v n}(S)\right\} \\
& \Gamma_{U}(\mathbb{N})=\neg\left(O_{v n}(\mathbb{N}) \vee O_{v n}(\neg \mathbb{N})\right)
\end{aligned}
$$

and so $\Gamma_{U}(\mathbb{N})$ is true. The complement partition of $\neg \mathbb{N}$ is given by:

$$
\begin{aligned}
& \neg \mathbb{N}=\left\{S \mid \Gamma_{U}(S) \wedge \neg O_{v n}(S)\right\} \\
& \Gamma_{U}(\neg \mathbb{N})=\neg\left(\neg O_{v n}(\neg \mathbb{N}) \vee \neg O_{v n}(\mathbb{N})\right)
\end{aligned}
$$

and so $\Gamma_{U}(\neg \mathbb{N})$ is false.

Since no set can contain a proper class, $U$ can only contain normal sets. One needs to be clear about what is meant by containment. Containment means a set has a sufficiently lower rank to be somewhere inside another set. While one can argue that $\neg \mathbb{N}$ is "contained" in $U$ in the sense that all its members are in $U$, that is a very different thing from saying $\neg \mathbb{N}$ is a member of $U$, which it isn't. $\neg \mathbb{N}$ is a partition of $U$ only. Every set is a partition of $U$, but not every set is a member of $U$. Since $U$ can only contain normal sets, the containment predicate of any set's comprehension can always be specified with respect to $U$ and Theorem 3.1 will always be satisfied. The use of $\Gamma_{U}()$ is more general and was implied earlier by the equivalence of a set as a partition of $U$. Note that $\Phi_{X}()$ in Theorem 3.2 made use of $\Gamma_{U}()$ instead of $\Gamma_{X}()$.

$U$ and $\emptyset$ can be made amenable to an inheritance analysis by defining $P_{t}(S)=$ true, $\forall S$ and $P_{f}(S)=$ false, $\forall S . \quad X$ in Theorem 3.2 can construct $U$ with $P_{x}()=P_{t}()$. Thus, $U$ is a proper class. Moreover $P_{t}(U)$ is true and so $U$ can be considered to inherit the property of its members, although the universality of the selection means it is only really inheriting, at best, the property of being a set. $X$ in Theorem 3.2 can construct $\emptyset$ with $P_{x}()=P_{f}()$. Thus, $\emptyset$ is not a proper class. Moreover $P_{f}(\emptyset)$ is false and so $\emptyset$ can be considered to not inherit the property of its members even though it has no members to inherit from. Even though $U$ and $\emptyset$ can be made amenable to an inheritance analysis, they are special cases. They do not really have any meaningful properties that can be inherited. In order for a property to have a proper heritable meaning it must partition $U$ into non-empty subsets with the least such division being into $U \backslash\{\emptyset\}$ and $\{\emptyset\}$ with respect to the property of having members. A heritable property is defined here as one which partitions $U$ into two non-empty subsets and a non-heritable property as one which does not.

The normal meaning of inheritance can be subverted by defining an inheritance property predicate, $P_{n}$, that constructs set $\mathrm{N}$, in terms of not inheriting as $P_{n}(X)=\neg P_{x}(X)$. Values of true and false for the property's own partition will have the opposite meaning to what they would normally have. A true value means that it inherits the property of not inheriting and 
so does not inherit. A false value means that it does not inherit the property of not inheriting and so inherits. Such a property is defined here as being an anti-inheritance property. The property is self contradictory if $X=N$ requires $x=n$. But if there is some $y \neq n$ with $Y=N$ then $P_{n}(N)=\neg P_{y}(Y)$. The complement property (a) that generates set $\mathrm{A}$ is not an anti-inheritance property and is never contradictory. The comprehension of both sets is

$$
\begin{aligned}
& \Phi_{N}(S)=\Gamma_{U}(S) \wedge P_{n}(S)=\Gamma_{U}(S) \wedge \neg P_{s}(S)=\neg \Phi_{S}(S) \\
& \Phi_{A}(S)=\Gamma_{U}(S) \wedge P_{a}(S)=\Gamma_{U}(S) \wedge P_{s}(S)=\Phi_{S}(S)
\end{aligned}
$$

$\Phi_{S}(S)$ is always false by Theorem 3.1 and so $N$ is $U$ and $A$ is the empty set. Thus, for the anti-inheritance property $\mathrm{n}$ :

$$
\begin{aligned}
& P_{n}(N)=P_{n}(U)=\neg P_{t}(U)=\text { false } \\
& P_{\neg n}(\neg N)=\neg P_{n}(\emptyset)=P_{f}(\emptyset)=\text { false }
\end{aligned}
$$

This is precisely the case with respect to the property of being normal. Returning back to the case of the modified Russell set:

$$
R^{\prime}=\left\{S \mid \Gamma_{U}(S) \wedge P_{N}(S)\right\}
$$

As stated earlier, one is free to separate out those sets that are normal from those sets that are abnormal. The $P_{N}()$ predicate will filter out the sets that are normal. For any set $\mathrm{X}$ with comprehension $\Phi_{X}(S)=\Gamma_{U}(S) \wedge P_{x}(S), P_{N}()$ will select those sets for which $P_{x}(X)$ is false. Thus, $P_{N}(X)=\neg P_{x}(X)$. So one can consider all possible partitions of $U$ and separate out those which are normal from those which are not. A small selection is shown below along with how the property of being normal was obtained.

\begin{tabular}{|c|cccccc|}
\hline$X$ & $\emptyset$ & $\mathbb{N}$ & $\mathbb{R}$ & $U$ & $\neg \mathbb{N}$ & $\neg \mathbb{R}$ \\
\hline$P_{N}(X)$ & $\neg P_{f}(\emptyset)$ & $\neg P_{\mathbb{N}}(\mathbb{N})$ & $\neg P_{\mathbb{R}}(\mathbb{R})$ & $\neg P_{t}(U)$ & $\neg P_{\neg \mathbb{N}}(\neg \mathbb{N})$ & $\neg P_{\neg \mathbb{R}}(\neg \mathbb{R})$ \\
& true & true & true & false & false & false \\
\hline
\end{tabular}

The set of all normal sets must be some such partition of $U$. If the property of being normal is a heritable property then this results in $P_{N}\left(R^{\prime}\right)=\neg P_{N}\left(R^{\prime}\right)$, which is the source of the paradox. However, since no set can contain a proper class, the set of abnormal sets is the empty set and so the modified Russell set is the set of all sets $\left(R^{\prime}=U\right)$. Thus $P_{N}\left(R^{\prime}\right)=P_{N}(U)=$ false. Note that since the property of being normal is an anti-inheritance property, a false value with respect to its own partition means that it does inherit the property of its members, which in this case is the property $P_{t}$. The property of being normal is a non-heritable antiinheritance property. The set of all sets that do not inherit from their members does inherit, but the property it inherits is not the property of not inheriting because the property of not inheriting is associated with every member of $U$ and so the set of all sets that do not inherit is just the set of all sets and the set merely inherits, at best, the property of being a set.

The framework of unrestricted comprehension appears to be consistent and free from contradictions. 


\section{The Diagonal Method}

The diagonal method is a method for constructing a new sequence that is not in the list of sequences spanned by the diagonal. To see it in operation consider the list of all possible sequences of three binary digits representing the numbers from 0 to 7 as shown in Figure 4.1

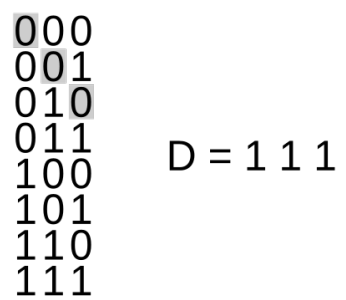

Figure 4.1: An example of a diagonal construction

The diagonal from the top left is highlighted. The method is to change each diagonal digit to any different valid digit. The result is that the diagonal sequence (D) cannot appear anywhere in the sequences covered by the diagonal, since it differs by at least one digit for every sequence in the list of items it covers. The diagonal sequence is in the list somewhere. It must be, in this case, since the list contains every possible sequence, but the method always produces a sequence that must be elsewhere in the list.

The diagonal method requires that there be the same or more rows than there are columns. If there are fewer rows, it cannot produce a sequence that is long enough to fully compare with any sequence in a row. If there are more rows than columns, there will be rows left over that the diagonal does not cover, which could potentially accommodate the value produced by the diagonal. If there are the same number of rows and columns, the diagonal covers the entire set of sequences. This is when the diagonal sequence becomes problematic and so can be used to show that no complete list of unique sequences can have the same number of rows and columns.

A well known use of the diagonal method is in Cantor's second proof of the uncountability of the reals [Cantor,1891]. If the reals are countable then a bijection can be found between the natural numbers and the real numbers. An example of a map is shown in Equation 4.1.

$$
\left[\begin{array}{ccccc}
0 & 0 & \cdot & 0 & 0 \\
0 & 0 & \cdot & 0 & 1 \\
0 & 0 & \cdot & 1 & 0 \\
0 & 0 & \cdot & 1 & 1 \\
\cdot & \cdot & \cdot & \cdot & \cdot \\
\cdot & \cdot & \cdot & \cdot & \cdot \\
1 & 1 & \cdot & 1 & 1
\end{array}\right][\mathbb{N}]=\left[\mathbb{R}^{b s}\right.
$$

The map operation is represented in matrix form (call it F), but it is not doing a standard matrix operation. Instead, it is placing a digit ( 0 or 1$)$ to the right of a decimal point at the same location it appears within the matrix row. The matrix rows contain every possible 
sequence of 1's and 0's and has the same form as the power set map. F can be constructed

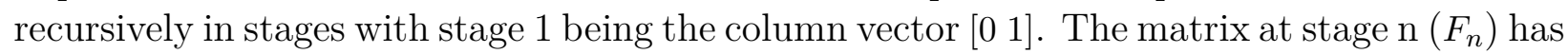
$2^{n}$ rows and $n$ columns. To construct stage $n+1$ make a copy of the rows below the existing rows and fill a new column to the left with $2^{n} 0$ 's followed by $2^{n} 1$ 's. Figure 4.1 shows $F_{n}$ at stage 3. The matrix in Equation 4.1 is a representation of $F_{n}$ as $n \rightarrow \infty$. The [N] column vector is a column of natural numbers with one entry for each natural number. The value of the natural number has no bearing on the output of the map and merely serves as a place holder for each natural number. The map maps to values within the range of the interval $[0,1]$ in binary decimal form, but it is not one-to-one. The image of $\mathrm{F}$ contains an infinite number of equivalent real values. For example, all continuous sets of sequences ending with

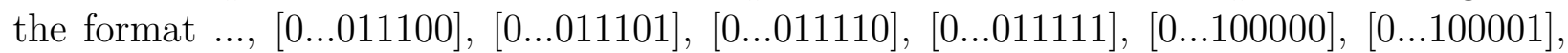
[0..100010], [0..100011] ... describe infinitesimally separated neighbouring values and thus evaluate to an equivalent real value. There are an infinite number of entries in each such sequence and each real is contained somewhere within each such sequence of which there are an infinite number. For another example, the second row of $\mathrm{F}$ maps to a value that is greater than zero and forever getting smaller beyond the precision of any real number since reals only have finite unbounded precision given by the set of well-founded ordinals. This is the smallest infinitesimal that can be represented by a single-infinite binary sequence. The map maps to the reals plus an infinite sequence representation of their infinitesimal neighbourhoods. This is labelled in Equation 4.1 as $\mathbb{R}^{b s} . \mathbb{R}^{b s}$ is the set of all infinite binary sequences. $\mathbb{R}$ is the subset of all well-founded binary sequences.

The map from $\mathbb{N}$ to $\mathbb{R}$ is obtained by removing the equivalent real value entries from $\mathbb{R}^{b s}$ to give $\mathbb{R}$. This will also remove rows from $F$ to give $F^{\prime}$. The resulting map from $\mathbb{N}$ to $\mathbb{R}$ in the interval $[0,1)$ is a subset of the power set map.

$$
\left[F^{\prime}\right][\mathbb{N}]=[\mathbb{R}]
$$

There are some technical issues as to how this can be achieved. For example, there is an issue as to what the second row should be, since there is no such thing as the first positive non-zero real number, so no single binary sequence can represent it. However, this is not relevant to the argument here. The key point here is that a great many rows need to be removed from $\mathrm{F}$.

Cantor's diagonal argument proceeds as follows, starting by assuming the matrix map from $\mathbb{N}$ to $\mathbb{R}$ is both square and complete. Taking the diagonal of the matrix and flipping each bit will produce a sequence that cannot be in any of the rows covered by the diagonal. But it must be, if it is square and complete, since the diagonal covers all possible rows and all possible rows are represented. Thus, there cannot be a bijection from the natural numbers to the real numbers. The cardinality of the reals must be greater than the cardinality of the natural numbers. This is a proof by contradiction in that it fails to explicitly show that the map is an injection. It merely assumes it must be, because assuming a bijection leads to an apparent contradiction. However, a bijection between a set and its power set will be constructed in Section 5, so there must be a floor in the argument somewhere. 
Cantor's diagonal argument has two solutions. Either the matrix is not square or it is not complete. The starting assumption that it is both square and complete is not valid, since the same argument can be used to show that no complete list of sequences can be square. The floor in the original argument is in starting with a configuration that is logically contradictory and thus guarantees a contradiction. If the matrix is not square, then, for infinite sets, the existence of a bijection does not require the matrix map to be square since there is no upper bound to the rows and columns. Some examples are given in Section 5. If the matrix is not complete, then there are rows missing for the diagonal to occupy. In the case of $F^{\prime}$, there are an infinity of rows missing. Thus, neither solution disproves a bijection.

The situation can be visualised by referring back to Figure 4.1. With each successive addition of a column (of a member to each sequence) the number of rows (sequences) doubles. However, there is no requirement for the diagonal to occupy successive rows or even be diagonal. All that is required is that each member of the diagonal occupy a unique row and column. Thus, the diagonal can be spread out over the rows. One may conjecture (assuming $|\mathbb{R}|=|\mathbb{N}|$ ) that each diagonal member could be arranged to map to a unique real with the gaps either side representing its infinitesimal neighbourhood. That may provide for a means to obtain $F^{\prime}$ from $F$ and solve the second row issue.

The nature of the infinitesimal neighbourhood can be seen with a version of Cantor's first uncountability proof [Cantor,1874]. If a number type is countable, then it can be indexed by sequence of natural numbers. Consider some ordered, continuous sequence of values that are indexed by a natural number as

$$
x_{l}, x_{l+1}, x_{l+2}, \ldots x_{u-2}, x_{u-1}, x_{u}
$$

in which $x_{l}$ is the lowest lower bound value and $x_{u}$ is the highest upper bound value. However, start off in this version with each $x$ value mapping to a row of $\mathrm{F}$. That is, the $x$ sequence is complete with respect to an infinite sequence representation.

The proof proceeds as follows. Assign $a_{1}$ to lowest bound of the sequence. Assign $b_{1}$ to the highest bound if not already assigned to $a_{1}$. This will form the closed interval $\left[a_{1}, b_{1}\right]$. Note that in this version the closed interval is formed one end at a time in a manner to prevent the possibility that both ends collapse to the same point. If the lower bound is closed, convert it to an open bound by excluding $a_{1}$ from the interval. Then, if the upper bound is closed, convert it to an open bound by by excluding $b_{1}$ from the interval. This creates the open interval $\left(a_{1}, b_{1}\right)$ with respect to the number type being considered. Now repeat, by taking the new lowest and highest bounds and collapse to form a new open interval at stage $\mathrm{n}$ given by $\left(a_{n}, b_{n}\right)$. Each collapse of one endpoint reduces the number of $x$ values in the interval by one. Eventually, even if after an infinite number of collapses, the two ends reach the point where no more collapses are possible. In the limit, $a_{n}$ tends to some point $x_{p}$ from below and $b_{n}$ tends to some nearest neighbour point $x_{p+1}$ from above. At this stage, every indexed $x$ value in the sequence has been excluded from the sequence. But, for real numbers, since the real number line is continuous, there must still exist values between the $x$ values, labelled here as $c$ values, with $c=\frac{1}{2}\left(x_{p}+x_{p+1}\right)$ as just one example, that cannot be contained in the indexed sequence. Thus, the real numbers cannot be indexed. 
However, in this version, the $x$ sequence is complete with respect to an infinite sequence representation. Every unique $x$ value maps to a unique infinite sequence row of $\mathrm{F}$ and visa versa. Thus, there is no new equivalent length infinite sequence that can represent $c$ since all such sequences are already allocated to an $x$ value. If $x_{p+1}=x_{p}+\delta$ for some infinitesimal separation $\delta$ represented by the sequence [0...1], then the middle value is given by $c=x_{p}+\frac{1}{2} \delta$. This offset is too small for a single-infinite binary sequence representation. That is, the resulting sequence is pushed beyond the precision of $\delta$ and into the precision of $\delta^{2}$. If $\delta=[0 \ldots 1]$ then $\delta^{2}=[0 \ldots 0 \ldots 1]$. Recall from Section 2 that one is free to subdivide an infinite sequence into infinite subsequences, which fixes the precision of a row of $\mathrm{F}$ and every row must have the same precision for each row to be comparable. For any finite choice of power of $\delta$, the $c$ value will be beyond the precision of such a choice.

If the infinitesimal neighbourhoods are removed from the $x$ sequence so that each $x$ value maps to a row of $F^{\prime}$, then while every real $x$ is mapped to a unique infinite sequence, every other sequence must not map to any real in order to maintain arithmetic consistency with respect to $c$. Thus, $c$ is not a real number but a value somewhere within the infinitesimal neighbourhood of the real number $x$ values. The closest real number to $c$ will either be $x_{p}$ or $x_{p+1}$ or both.

The essential nature of the argument is that, for any starting assumption that the $x$ sequence is countable and complete, with respect to some property, then the $c$ value can never have that property, because the sequence is always assumed to be complete with respect to being countable. Every possible countable value is in the sequence. So if the $c$ value does have the same property, then the sequence cannot be countable. Cantor's original argument starts by assuming that the $x$ sequence is complete with respect to a countable sequence of reals, but it only works if there are values in-between the $x$ values that are assumed to be a real number, for then there are some reals that are not in the countable sequence. If instead, one insists the sequence is countable, then it actually proves the existence of numbers that are not real that lie between the reals. Cantor's proof has two solutions. For any $c$ value, if $c$ is real, then the reals cannot be counted, but if the reals can be counted, then $c$ cannot be real. The issue of uncountability did not arise for the cases of the $x$ values representing rows of $F$ or $F^{\prime}$ since in those cases the $c$ value was manifestly of a different number type.

The proof of the countability of all sequences is in Section 5, which shows that the set of all cardinals is equivalent to the set of natural numbers. Since all sequences are countable, Cantor's first uncountability proof results in an infinite descending hierarchy of scales. For any continuous number type there is another continuous number type embedded between its values, associated with the next power of $\omega$ when represented as an infinite list embedded between each item of another infinite list. This can be seen in the expression for $\delta^{2}$ above. Thus, not only are there infinitesimals, but also infinitesimals of infinitesimals and so on. This justifies the Leibniz method of calculus.

Cantor's proof implicitly assumes, and can only be carried out if, there exists a diagonal map from $\mathbb{N}$ to the $x$ sequence, since each elimination of an $x$ value removes both one row and one column from the map. Such a map is always possible, for a countable sequence, by virtue of the index on the sequence value acting as the map. Since the reals are countable, 
this confirms the earlier conjecture of arranging for each diagonal member of $F$ to map to a real number, which results in $F^{\prime}$ being square. From which it then follows, by Cantor's diagonal argument, that the matrix rows for $F^{\prime}$ must not be complete.

Given that $F^{\prime}$ is square, then any $c$ value is the value that the diagonal method finds, by constructing a sequence different from every sequence it extracts a member from. It extracts a member (from a row of $F^{\prime}$ ) from every sequence matching one of the real $x$ values and constructs a sequence that, by design, is not a sequence representing any of the real $x$ values.

The above undermines the use of the diagonal method to produce a sequence not in a given list and then claim that such a result is contradictory, since the diagonal method, by design, always finds a sequence that does not belong in the list it is selecting members from. Any such attempt would have to first demonstrate that (1) the list of sequences it is selecting members from is complete and (2) the sequences have the same number of rows and columns. However, items 1 and 2 are mutually exclusive. There is no complete list of sequences that has the same number of rows and columns and the diagonal method is precisely the method that proves this. The diagonal method fails on $[F][\mathbb{N}]=\left[\mathbb{R}^{b s}\right]$ because $F$ is not square and fails on $\left[F^{\prime}\right][\mathbb{N}]=[\mathbb{R}]$ because $F^{\prime}$ is not complete.

Understanding whether something is or is not a diagonal method is critical to understanding what is a valid assignment for what the method produces. Diagonal methods are more varied than the matrix method considered here. Some other types are considered in the next section. A diagonal method is any procedure that produces an object comparable to, but different from, every object it samples over. Consequently, the diagonal construction must never be set equal to any of the objects that were sampled, since that will create a contradiction. It can be made to work with any collection of objects that are composed of common parts in which some parts have multiple possibilities, which may or may not involve the part being present. The number of permutations is at a minimum when each modifiable part has only 2 possibilities. But even in that case, for $n$ parts there are $2^{n}$ permutations. So a complete enumeration of objects always contains more objects than there are parts to modify within each object. It is typically applied to sequences, constructing sequences from modified parts of sampled sequences and, in general, proves that more sequences are possible than those that can be sampled in a single diagonal construction. A single diagonal construction can never sample every possible sequence.

Since the proof against a bijection between the natural numbers and the real numbers fails, this leaves open the possibility that a bijection can be found between the natural numbers and the real numbers. This will be addressed in the next section. 


\section{Cantors's Paradox and Cantor's Theorem}

Cantor's paradox is that the set of all cardinals is a contradictory set. It is related to the Burali-Forti paradox, since the cardinality of a set is the least ordinal number such that there is a bijection between the set and the ordinal. So it is always possible to find an ordinal that corresponds to a cardinal. Specifically, the set of all cardinals is a subset of the set of all ordinals. Since the set of all ordinals was shown to be non-contradictory in Section 2, the set of all cardinals should also be non-contradictory.

But this has other implications, for it is possible to arrive at Cantor's paradox by other means, specifically through the application of Cantor's theorem. Suppose there is such a thing as the set of all cardinals, $C$. The set of all cardinals is not itself a cardinal in the same way that the set of all ordinals is not an ordinal. But it is a set and so it is possible to take the power set of this set, $P(C)$. By Cantor's theorem

$$
|P(C)|>|C|
$$

which means that the cardinality of the the power set is greater than the cardinality of any value in the set of all cardinalities, which is a paradox. It implies there cannot be a set of all cardinalities. But there must be, since there is a set of all ordinals and every cardinal is somewhere in the set of all ordinals. Cantor's theorem cannot be correct.

Cantor's theorem is typically proved with the following argument [Hessenberg,1906]. For any set, it is always possible to define a map $(f)$ between the set and its power set: $f: S \mapsto P(S)$. For a set $S$ with members $s_{1}, s_{2}, \ldots$ there is always at least the map $f\left(s_{i}\right)=\left\{s_{i}\right\}$. For any finite set, there will be members of the power set missing from the image of the map and so the map is at least an injection. That is, there are never less members in the power set. But for infinite sets it is possible for sets with differing degrees of indexable members, depending on their ordering, to have the same cardinality, since the cardinality is independent of any ordering. If the cardinality of the power set is the same as its source set then $f$ must be a bijection. Each endpoint $f\left(s_{i}\right)$ is a set that contains some number of members of $S$. The endpoint may or may not contain the set member it was mapped from. Now construct a set $(T)$ of all those $s_{i}$ members that are not members of the endpoint of their map

$$
T=\{x \in S \mid x \notin f(x)\}
$$

Since $\mathrm{T}$ is a set containing some number of members it must be one of the members of the power set and since $f$ is a bijection, there must be some $y \in S$ such that $f(y)=T$. Now consider if $y$ is in $T$. If $y$ is in $T$ then it satisfies $y \notin f(y)$ and so is not in T. Conversely, if $y$ is not in $T$ then it satisfies $y \in f(y)$ and so is in T. This is a logical contradiction which suggests there is no such bijection. This is another proof by contradiction.

Notice that the set up involves a self reference via a negation. In words "The map of $\mathrm{y}$ is equal to the set $(\mathrm{T})$ of all members that do not contain themselves in their map". Considering if $\mathrm{y}$ is in $\mathrm{T}$ creates a self reference to the negation of the rule for being in $\mathrm{T}$ and guarantees a paradox. Since the process described can be physically carried out, there 
must be something in the way the process works that negates the paradox. One way to visualise what is physically happening is by re-framing it as a diagonal method. The map $f$ can be written in matrix form $F$ as shown in Equation 5.3, in which the matrix coefficients determine which members of $\mathrm{S}$ are members of each of their end points.

$$
\left[\begin{array}{ccccc}
0 & 0 & \cdot & 0 & 0 \\
0 & 0 & \cdot & 0 & 1 \\
0 & 0 & \cdot & 1 & 0 \\
0 & 0 & \cdot & 1 & 1 \\
\cdot & \cdot & \cdot & \cdot & \cdot \\
\cdot & \cdot & \cdot & \cdot & \cdot \\
1 & 1 & \cdot & \cdot & 1
\end{array}\right]\left[\begin{array}{c}
s_{1} \\
s_{2} \\
\cdot \\
\cdot \\
\cdot
\end{array}\right]=\left[\begin{array}{c}
f\left(s_{1}\right) \\
f\left(s_{2}\right) \\
f\left(s_{3}\right) \\
f\left(s_{4}\right) \\
\cdot \\
\cdot \\
\cdot
\end{array}\right]
$$

The mapping matrix contains rows with every possible unique sequence of zeros and ones. It is the same F matrix as in Section 4 except that now it is applied in a different way and there are no equivalent values being mapped to. The row for the map for the $T$ set can be obtained by taking the top-left diagonal of $F$ and flipping each of the digits. Referring back to Figure 4.1, with each successive addition of a column the number of rows doubles. Thus, there are plenty of rows below the diagonal for the sequence produced by the diagonal to occupy. The $\mathrm{T}$ sequence must be one of those rows. Now consider if $y$ is in $T$. That is, is there a 1 in the $T$ row and $y$ column. It will not be possible to know this until later stages in the construction of $F$, since the relevant $y$ for any given row is contained within the diagonal and so the $y$ for the potential $T$ row does not yet exist. But, by the time the diagonal reaches any given target row, the $T$ row must have moved further down, since, by design, the row the diagonal constructs is always different to every row contained within the diagonal. Consequently, it is easier to see how the contradiction in the Hessenberg proof is avoided by this particular map, since it is not square. As a result, the $T$ set can obviously, as it must, forever track below the diagonal during its construction and be mapped to, from a $y$ in which the the decision about whether $y$ is in $T$ has not been made, so no contradiction arises. The error in the proof, with respect to this map, lies in the assumption that $T$ in Equation 5.2 can be completed when, in fact, there is always some $y \in S$ that is beyond $x \in S$. This is because the map is not square. The arrangement is shown later on in Figure 5.1. Although $T$ cannot be completed, its limit may be predicted if it has a predictable pattern. For example, if $F$ is constructed by always adding columns to the right, then, for a top-left continuous diagonal, every value along the diagonal will be zero and so $T$ tends to $S$.

The above map is not the only way to represent the power set map. If a bijection exists, then it is always possible to construct a diagonal version of the map between each $s_{i}$ member of $S$ and each $M_{i}$ member of $P(S)$ given by $f\left(s_{i}\right)=M_{i}$ and shown in matrix form in Equation 5.4.

$$
\left[\begin{array}{ccccc}
M_{1} & 0 & \cdot & 0 & \cdot \\
0 & M_{2} & \cdot & 0 & \cdot \\
\cdot & \cdot & \cdot & \cdot & \cdot \\
0 & 0 & \cdot & M_{n} & \cdot \\
\cdot & \cdot & \cdot & \cdot & \cdot
\end{array}\right]\left[\begin{array}{c}
s_{1} \\
s_{2} \\
\cdot \\
s_{n} \\
\cdot
\end{array}\right]=\left[\begin{array}{c}
M_{1} \\
M_{2} \\
\cdot \\
M_{n} \\
\cdot
\end{array}\right]
$$

The $s_{i}$ values in the column vector do not affect the output of the map, whose rule is to 
output the diagonal member from the same row. For this map, there is no $y \in S$ that is beyond $x \in S$. But, since all representations are equally valid, they must be equally possible. Applying the Hessenberg proof to this map is equivalent to locating some $n^{\prime}=n$ for $T=\left\{s_{i} \mid s_{i} \notin M_{i}\right\}$ such that

$$
f\left(s_{n^{\prime}}\right)=M_{n^{\prime}}=T=\left\{s_{1} \notin M_{1}, s_{2} \notin M_{2} \ldots, s_{n} \notin M_{n}, \ldots\right\}
$$

where $s_{n} \notin M_{n}$ is shorthand for only placing $s_{n}$ in $T$ if the logical expression is true. But since $M_{n}$ is the same as $T$, this results in the rule for being added to $T$ as being the negation of itself, which is the source of the contradiction. Using the naming syntax from earlier, the problematic map item is

$$
f(y)=T=\left\{s_{1} \notin M_{1}, s_{2} \notin M_{2} \ldots, y \notin T, \ldots\right\}
$$

So if $y$ is not in $T$, then it is in $T$ and visa versa. This does not prove that the map is impossible. It only proves that $y$, being inside the map, is impossible. The contradiction is avoided if, or rather implies that, there is no such $y$ that satisfies Equation 5.6. During the construction of $T$, the contradiction only exists if $y$ is set to one of the members already sampled by the partial construction of $T$, which are those that lie within the diagonal and for which it has already been determined whether or not that member is in $T$. Thus, in the context of a partial completion, the same contradiction can be used to show that $y$ cannot be any such member and so $y$ forever tracks below the diagonal during its construction. Equally, $T$ can never be a member of $P(S)$ at any stage of construction due to being different from every $M_{i}$ member of $P(S)$ via the diagonal method in Equation 5.5. The arrangement is shown later on in Figure 5.2. The error in the Hessenberg proof, for this map, is in the assumption that $T$ is a member of $P(S)$, but no sequence generated by the diagonal method can ever be equal to any of the sequences it sampled. A concrete example for this map is given later on in Equation 5.14. The ultimate source of the contradiction in the Hessenberg proof, for any map, lies in setting a sequence generated by the diagonal method equal to one of the sequences it sampled.

As a historical aside, one can see how Russell obtained the Russell set from an analysis of Cantor's theorem by considering $\mathrm{S}$ to be the universal set of all sets $(U)$ and $f$ to be the identity map $f(x)=x$. Then the T set becomes $T=\{x \mid x \notin x\}=R$. If $\left\{m s_{n}\right\}$ is the set of all members of the $s_{n}$ member of $S$, then Equation 5.5 applied to the identity map results in the statement that there is no such $n$ that will satisfy

$$
\left\{m s_{n}\right\}=T=\left\{\ldots, s_{n} \notin\left\{m s_{n}\right\}, \ldots\right\}
$$

which is an alternative statement of the Russell paradox. There is only the off diagonal solution for some unmapped set, indexed by $n^{\prime}>n$, with $n$ being the extent of the diagonal

$$
\left\{m s_{n^{\prime}}\right\}=T=\left\{s_{1} \notin\left\{m s_{1}\right\}, s_{2} \notin\left\{m s_{2}\right\}, \ldots, s_{n} \notin\left\{m s_{n}\right\}\right\}
$$

$T$ is only composed of sets that do not contain themselves and is specifically designed, by the diagonal method, to be different from every member contained within the diagonal and so never contains itself and forever tracks below the diagonal. In the limit, $T$ is composed of 
every set that does not contain itself and yet none of those sets can be $T$. The contradiction can only be avoided if no set can contain itself. It cannot be solely avoided by $T$ being a proper class as defined in Section 3, such that $T$ only contains containable sets, since that definition depends on no set containing itself. It could be avoided by declaring $T$ not to be a set, but that would be subject to the same circularity criticisms of Section 3. If the $\notin$ operator in Equation 5.8 is replaced with an operator that returns true if a set is not contained anywhere inside the containment hierarchy of some given set, then the conclusion will be the same with respect to that operator. Thus, no set can be contained anywhere within its own containment hierarchy and so $T=R=U$.

It remains to show that a bijection can be constructed between an infinite set and its power set. The power set members of some set $S$ having members $\left\{s_{1}, s_{2}, \ldots\right\}$ can be enumerated with the following algorithm: starting with the empty set as the first member, form a seed set $\left(S_{s}\right)$ containing an unused member from $S$ and create new members of the power set by taking the union of the seed set with each of the previously existing members. So, for example, the members of the power set of $\{1,2,3\}$ are enumerated as

$\begin{array}{lll} & & \{\} \\ S_{1}=\{1\} & \{1\} \cup\{\} & \{1\} \\ S_{2}=\{2\} & \{2\} \cup\{\} & \{2\} \\ & \{2\} \cup\{1\} & \{1,2\} \\ S_{3}=\{3\} & \{3\} \cup\{\} & \{3\} \\ & \{3\} \cup\{1\} & \{1,3\} \\ & \{3\} \cup\{2\} & \{2,3\} \\ & \{3\} \cup\{1,2\} & \{1,2,3\}\end{array}$

The number of members generated by each successive seed count $s$ is equal to the count of all the previous members and is given by $2^{s-1}$. Define the following:

- $S_{s}$ is the seed set containing the s'th member of $\mathrm{S}: S_{s}=\left\{s_{s}\right\}$

- $M_{i}$ is the i'th member of $P(S)$

- $m_{s i}$ is the i'th member generated by the seed set $S_{s}$

The members of the power set, of a set with $n$ members, are then generated by

$$
\begin{aligned}
& M_{1}=\{\} \\
& M_{2^{(s-1)}+i}=m_{s i}=S_{s} \cup M_{i} \quad \text { if } n \geq 1 \text {; for } s=1 \text { to } n, i=1 \text { to } 2^{s-1}
\end{aligned}
$$

The bijection from $S$ to $P(S)$ is from $s_{i}$ to $M_{i}$, where $i$ is any member of $\mathbb{N}$ and so includes infinite sets and infinite sets of infinite sets and so on as shown in Section 2. Thus, for any infinite set, its cardinality is equal to the cardinality of its power set and Cantor's Theorem needs to be modified as

$$
|P(S)| \geq|S|
$$

with the equality sign applying to any infinite set. This eliminates Cantor's paradox. 
The cardinality of the real numbers cannot be less than the cardinality of the natural numbers and the cardinality of any subset of the power set cannot be greater than the cardinality of the power set. The map between $\mathbb{N}$ and $\mathbb{R}$ is a subset of the power set map of $\mathbb{N}$ and so the cardinality of the reals is the same as the cardinality of the natural numbers, which also proves the continuum hypothesis.

$$
|\mathbb{R}|=|\mathbb{N}|
$$

The Von Neumann universe is built from the ground up from the empty set. Every set is a subset of successive power sets of the empty set. In such a universe there is only one infinite cardinality and the set of all cardinals is equivalent to the set of all natural numbers. This must be the case if $\Omega=\mathbb{N}$, since the set of natural numbers is the only counting set that there is.

The existence of a single infinite cardinal count does not mean that all infinite matrices are square. Consider indexing the rows and columns of a matrix by a diagonal count. Consider also that each diagonal position is marked by some indistinguishable marker. The row count for a non-square infinite matrix with more rows than columns can only be fully symbolically indexed by additional transfinite ordinals. Even if there is just one more row than the number of columns, it is not possible to re-order and re-index the row list without throwing away the relative indexing of the rows and columns and starting again with a new diagonal count. If instead, the original diagonal markers are retained and the relative indexing is ignored and the rows are re-ordered and re-indexed only up to $\omega$ instead of $\omega+1$, there will not be sufficient indices to label all the rows, since the row count will then be the same as the marker count and yet there is never more than one marker in any row and there is always one row that does not contain a marker. Thus, it is not possible to abstract away the size of any matrix into two independent cardinal counts. Matrices are ordered structures whose relative size must be described using ordinals, or, for greater flexibility, some number type capable of describing fractions of infinite quantities, such as $\frac{1}{2} \omega, \log _{2} \omega$ or even $\omega-1$. The motivation for such a number type is in the fact that the vectors a matrix operates with can be separately given an independent cardinal count. It is only the relative size of such vectors that cannot be described by cardinal counts, or rather, the relative cardinal count has a different meaning. It describes the relative countability of each sequence or the relative ultimate count of each sequence.

A common feature of both Cantor's diagonal argument and the Hessenberg proof of Cantor's theorem is an assumption that the matrix is square, but the mere fact that the matrix is infinite in size is not sufficient justification for it to be considered square. The reasoning behind the assumption of a square matrix is linked to the idea that a bijection involves a square matrix. While a square map can always be found, the existence of equally valid non-square alternatives requires that such alternatives be equally valid bijections. How can there be a bijection between vectors of different lengths? A bijection is possible because the lengths are infinite. Infinity is not a static value, but a process of forever getting larger. The shorter vector is forever moving into the space needed to keep the bijection going while the larger vector is forever ahead of the shorter. Since the process never ends, the bijection is sustained. 
One example is a map between the odd natural numbers and the full set of natural numbers that is shown in each direction in Equation 5.13

$$
\left[\begin{array}{ccccccc}
0 & 1 & 0 & 0 & 0 & 0 & . \\
0 & 0 & 0 & 1 & 0 & 0 & . \\
0 & 0 & 0 & 0 & 0 & 1 & . \\
\cdot & \cdot & \cdot & \cdot & \cdot & \cdot & \cdot
\end{array}\right]\left[\begin{array}{l}
0 \\
1 \\
2 \\
3 \\
4 \\
5 \\
\cdot
\end{array}\right]=\left[\begin{array}{l}
1 \\
3 \\
5 \\
\cdot
\end{array}\right] \quad, \quad \frac{1}{2}\left[\begin{array}{cccc}
0 & 0 & 0 & \cdot \\
2 & 0 & 0 & \cdot \\
1 & 1 & 0 & \cdot \\
0 & 2 & 0 & \cdot \\
0 & 1 & 1 & \cdot \\
0 & 0 & 2 & . \\
\cdot & \cdot & \cdot & \cdot
\end{array}\right]\left[\begin{array}{l}
1 \\
3 \\
5 \\
\cdot
\end{array}\right]=\left[\begin{array}{l}
0 \\
1 \\
2 \\
3 \\
4 \\
5 \\
\cdot
\end{array}\right]
$$

Other maps are possible, but these particular matrices are not square. They cannot be, since half of all rows or columns will never contain a diagonal marker. If the longer vector has length $\omega$, then the shorter vector has length $\frac{1}{2} \omega$. Since there is only one infinite cardinal, the cardinal count for both values is $\aleph_{0}$. The middle indices of the vectors (for just one example) are tending to infinity just as much as the last and there is only one infinity that can be tended to, since infinity is not a number, but a process that never ends.

Notice that if the non-square map from the odd naturals to the complete set is indexed by the diagonal, the whole set will have to be indexed up to $\omega 2$. The implication of this is that transfinite ordinals have no absolute meaning. They are an optional way (see Section 2) to impose some hierarchical structure on infinite sequences that may provide some useful organizational features. But ultimately, since there is only one infinite cardinal, the same sequence can be re-indexed with natural numbers and any comparative sequence must be rescaled consistently. All infinite sequences are countable and can be placed in a bijection with each other. All non-cardinal infinite values are relative measures only, of progress towards the same, unreachable, cardinal, destination.

For the non-square power set map, if $P(S)$ scales as $\omega$, then $S$ scales as $\log _{2} \omega$. The arrangement described earlier along with the relative sizing is shown in Figure 5.1.
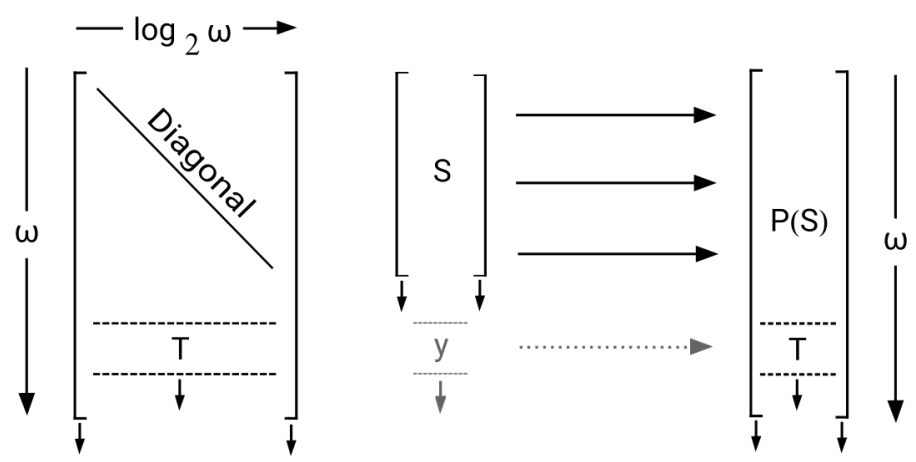

Figure 5.1: The relationship between non-square power set map items

Any current $y$ will eventually be consumed by $S$, but by then $y$ will have moved further down and will never be a member of $S$. For the power set of $U, P(U)$ maps to $U$, which is most easily seen by constructing $F$ by always adding columns to the right. The current $U, T$ and $P(U)$ are all tending to $U$, with $P(U)$ always further ahead and containing the $U$ that produced it and so $U$ is forever trying to contain itself and never succeeding. All of 
the sequences below the diagonal are tending towards proper class sequences due to being outside $U$, but they can never get there due to being inside $P(U)$. As stated earlier, $T$ can never be completed, but it may be predicted. The limit of $T$ is not fully amenable to a static interpretation due to $y$ being outside the map and $T$ being inside the map. Non-square bijections are inherently dynamic. Square maps are easier to work with. For the diagonal version of the power set map, if $P(S)$ scales as $\omega$, then $S$ also scales as $\omega$. The arrangement described earlier along with the relative sizing is shown in Figure 5.2.
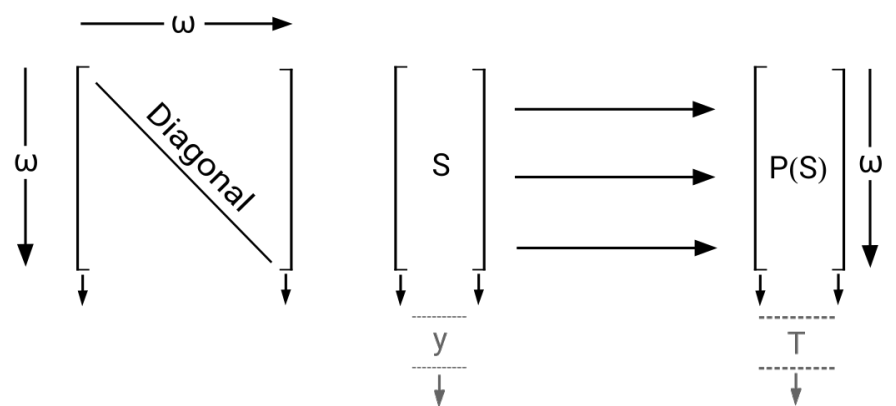

Figure 5.2: The relationship between diagonal power set map items

Note that since both $y$ and $T$ are below the rows of $S$ and $P(S)$, they are never members of their respective $S$ and $P(S)$. For example, for the power set of $\mathbb{N}, T$ can be arranged to tend to $\mathbb{N}$ and yet must be different from every member of $P(\mathbb{N})$, which should also contain $\mathbb{N}$. However, while both some non-fixed member of $P(\mathbb{N})$ and $T$ are tending to $\mathbb{N}, T$ is always further ahead and tends to being infinitely further ahead, by a factor of $\omega / \log _{2} \omega$. A partial construction for the first 8 members, generated using Equation 5.10, is shown in Equation 5.14, with $T$ being generated by Equation 5.5.

$$
\begin{array}{lll}
s_{1}=0 & M_{1}=\{\} & \\
s_{2}=1 & M_{2}=\{0\} & \\
s_{3}=2 & M_{3}=\{1\} & \\
s_{4}=3 & M_{4}=\{0,1\} & y=s_{256} \\
s_{5}=4 & M_{5}=\{2\} & T=\{0,1,2,3,4,5,6,7\} \\
s_{6}=5 & M_{6}=\{0,2\} & \\
s_{7}=6 & M_{7}=\{1,2\} & \\
s_{8}=7 & M_{8}=\{0,1,2\} &
\end{array}
$$

Notice that $s_{256}$ does not currently exist and by the time it does exist, $T$ will have moved further down. $M_{2^{n}}$ and $T$ are tending to the same limit but are never the same sequence. Consequently, two infinite sequences cannot be fully compared solely in terms of their limit. One sequence is always further ahead than the other, represented by their relative lengths of $\omega$ vs $\log _{2} \omega$. An example of a failure to take this into account is in the standard proof that $0.9999 \ldots$ is the same as 1 . Consider, the proof for a finite sequence

$$
\begin{array}{ll}
x & =0.9999 \\
10 x & =9.999 \\
9 x & =10 x-x=8.9991 \\
x & =9 x / 9=0.9999
\end{array}
$$


No matter how many 9's there are in the sequence, the result is the same. In the infinite limit, if $0.9999 \ldots$ contains $\omega$ digits after the decimal place then after multiplying by 10 it contains $\omega-1$ digits after the decimal place. An infinite sequence is simply a finite sequence that is forever growing in size. Whatever logic or arithmetic that applies to a finite sequence of any length must also apply to its infinite extension. The standard proof conjures up an additional digit for $10 x$ from nowhere so that the subtraction evaluates to 9 . The number values of $0.9999 \ldots$ and 1 are equivalent real numbers because they are separated by an infinitesimal in the infinite extension of $0.9999+0.0001=1$, but they are never the same number because an infinitesimal is never zero. Similarly, an exact finite decimal representation of $\frac{1}{9}$ to four decimal places, obtained by long division, is $\frac{1}{9}=0.1111 r 1=0.1111+\frac{0.0001}{9}$. Extending the sequence indefinitely does not eliminate the remainder; it is never zero. So multiplying by 9 results in 1, for any length of sequence.

The square diagonal version of the power set map of $U$ maps to $U$, since $U$ can be constructed with the recursive relation $U_{n+1}=U_{n} \cup P\left(U_{n}\right)$ with $U_{0}=\{\}$. This is the same as $U_{n+1}=P\left(U_{n}\right)$ since for a $U$ built from the empty set the power set reproduces all of the current members. So one is free to choose whether or not to always retain the current $U$ during its construction. A partial construction of the first eight members, generated using Equation 5.10, is show in Equation 5.16.

$$
\begin{aligned}
& s_{1}=M_{1}=\{\} \\
& s_{2}=M_{2}=\left\{s_{1}\right\} \\
& s_{3}=M_{3}=\left\{s_{2}\right\} \\
& s_{4}=M_{4}=\left\{s_{1}, s_{2}\right\} \\
& s_{5}=M_{5}=\left\{s_{3}\right\} \\
& s_{6}=M_{6}=\left\{s_{1}, s_{3}\right\} \\
& s_{7}=M_{7}=\left\{s_{2}, s_{3}\right\} \\
& s_{8}=M_{8}=\left\{s_{1}, s_{2}, s_{3}\right\}
\end{aligned}
$$

While some non-fixed member of $P(U)$ is tending to $U$, it is also tending to being infinitely further behind $T$, defined by Equation 5.5, which is also tending to $U$. The contained $U$ is always smaller than the current $U$, given by $T$, which is never a member of the current $U$. $U$ is forever trying to contain itself through successive power sets, but never succeeds.

Although the power set map can be arranged into a square form, the sequences within the diagonal members still have the structure of Figure 5.1 and so the diagonal can never sample every possible sequence, even in the infinite limit of sampling every possible member of the map, since many more possible sequences lie outside the diagonal, in either version of the map. Any sequence produced by a diagonal method on the diagonal power set map of $U$ must be a proper class, since it is not a member of $U$. In the limit, $T$ tends to $U$ and so contains every containable set and is not itself a containable set and so the limit is logically self consistent. Equation 5.5 is just one possible diagonal method. Any property that $T$ inherits will work as a diagonal rule for the power set/identity map of $U$ (i.e. $s_{i}=M_{i}$ ) with $T=\left\{s_{i} \mid P_{p}\left(s_{i}\right)\right\}$ provided $P_{p}(T)$ is true. This is because any possible $T$ inside $U$ could only be set to an $s_{i}$ that does not inherit the same property or else it would belong in $T$. So all sets that inherit the property of their members are proper classes. Consider the rule 
$T=\left\{s_{i} \mid s_{i} \notin s_{t}\right\}$ for all $i$ and some fixed $s_{t}$. This will also produce a set different from every possible $s_{i}=M_{i}$ member of $U$, since it contains $s_{t}$ and so inherits the property of not being a member of $s_{t}$. Thus, there is no $s_{i} \in U$ that can equal $T$. In the limit, $T$ tends to the complement of $s_{t}$ and contains $s_{t}$ and inherits the property of its members. Thus, the complement of every given member of $U$ is a proper class that inherits the property of its members and which contains the given member. Consequently, every member of $U$ does not inherit the property of its members.

For a fully square map there will always be many sequences that are more clearly outside map. For example, for the identity map of $\mathbb{N}$ in the form of Equation 5.4 and shown in Equation 5.17, the $T$ set for this map, as defined by Equation 5.5, tends to $\mathbb{N}$ and $\mathbb{N}$ is just one of many sequences of natural numbers that is not contained within the image of the map, which only contains continuous sequences. In this case, the diagonal is acting on an object in which each part is either present or not present.

$$
\begin{aligned}
& s_{1}=0=M_{1}=\{\} \\
& s_{2}=1=M_{2}=\{0\} \\
& s_{3}=2=M_{3}=\{0,1\} \\
& s_{4}=3=M_{4}=\{0,1,2\} \\
& s_{5}=4=M_{5}=\{0,1,2,3\} \\
& s_{6}=5=M_{6}=\{0,1,2,3,4\} \\
& s_{7}=6=M_{7}=\{0,1,2,3,4,5\} \\
& s_{8}=7=M_{8}=\{0,1,2,3,4,5,6\}
\end{aligned}
$$

If a fixed member of the map is chosen, given here by $\alpha$, then in the infinite limit $\alpha+1$ always exists for any choice of such an $\alpha$. This is a definition of a limit ordinal in the standard approach, but it is not unique to ordinals. Any infinite sequence will have this property. In the standard approach, infinite successor ordinals are still infinite sequences, but with the infinite part hidden away inside a symbol. When written out in long form, there is nothing to distinguish the limit of $\omega$ from $\omega+1$, which is given by $S^{+}(\mathbb{N})$ in Section 2 . In the approach here, limits also apply to finite sequences, which must be the case, since an infinite sequence is considered to be a finite sequence that is forever growing and so whatever logic applies to a growing finite sequence must also apply to its infinite extension and visa versa. For the finite construction in Equation 5.17, the map is a map for $\mathbb{N}_{7} . T=\mathbb{N}_{7}$ is not an ordinal of the current map, since it is not a member of the current map of ordinals. It is a limit ordinal-sequence for the current map, precisely because it is not an ordinal. In the infinite limit $T \rightarrow \mathbb{N}$, which is similarly not an ordinal and is a limit ordinal-sequence, for the same reasons. A limit ordinal-sequence, as defined in Section 2, is unique to the way ordinals are defined and not a general feature of sequences, whether finite or infinite. Note that the last member of the map is never equal to $T$, but they are both tending to $\mathbb{N}$, as are an infinite number of non-fixed members, when selected as some fraction of the current list length, down to a minimum location greater then $n^{1 / n}$ for list length $n$ as $n \rightarrow \infty$. The last member and $T$ are related by $T=S^{+}\left(s_{n}\right)$ or $s_{n}=S^{-}(T)$ for all $n$ as $n \rightarrow \infty$. In the infinite limit both sequences are tending to $\mathbb{N}$ and so can be represented by $\omega$ and are related to each other by $\omega+1$ and $\omega-1$. 


\section{Semantic Diagonalization}

Semantic diagonalization is a process that engineers a situation in which an object becomes the negation of itself and so violates the law of identity and the law of non-contradiction. This should be sufficient to invalidate the process, except that it can be obtained by processes that can in principle be physically carried out and so is the source of a great many paradoxes. However, this just means that something about the way a particular process works must end up invalidating the contradiction. To see it in operation, consider the proof of Cantor's theorem discussed in Section 5. It involves a map from a set, $S$, to its power set. Each map item maps from a member of $S$ to a set containing various members of $S$ and it is clear that the end point of the map may or may not contain the member it was mapped from. The $T$ set is constructed as the set containing all those members that are not in the end point of their map item. If $f(x)$ is a specific map item for $x$ and $P_{T}(x)$ is the predicate that $x$ is a member of the $T$ set, then

$$
P_{T}(x)=\neg(x \in f(x))
$$

This involves an expression that contains a free variable $x$ that ranges over every member of $S$. The proof considers that if there is a bijection then there is some $y \in S$ that maps to $T$ with $f(y)=T$. Semantic diagonalization involves setting the free variable to a specific value that induces a self reference. Considering if $y$ is in $T$ produces

$$
P_{T}(y)=\neg(y \in f(y))=\neg(y \in T)=\neg P_{T}(y)
$$

Thus, the rule for considering if $y$ is in $T$ becomes the negation of itself. The resolution, in this case, is that the $\mathrm{T}$ set forever tracks below the diagonal and so there is no $y$ that exists within the map.

Richard's paradox [Richard,1905] has numerous variations. The original version involved a map from the natural numbers to statements defining real numbers. This version considers a map from the natural numbers to any statement about the natural numbers. A particular map item may or may not map a natural number to a statement that the natural number belongs to. For example, if 2 maps to the statement "odd numbers are not divisible by 2 " then the number 2 does not belong to the numbers described by the statement. If the number 3 maps to the statement "prime numbers are numbers only divisible by one and themself", then the number 3 does belong to the numbers described by the statement. This has an identical character to the proof of Cantor's theorem. A Richardian number is defined to be a number in the domain of the map that does not belong to the numbers described by the statement it maps to. If $P_{R}(x)$ is the predicate that $x$ is a Richardian number and $f(x)$ is a specific map item for $x$ and $T(x, f(x))$ is a test function that tests if the number in the first argument is described by the statement in the second argument, then

$$
P_{R}(x)=\neg T(x, f(x))
$$

As before, the expression contains a free variable that ranges over the domain of the map. Since the map contains every possible statement about the natural numbers it should contain 
a definition of a Richardian number. If $y$ maps to the statement defining a Richardian number then $T(y, f(y))$ is true if $y$ is Richardian and semantic diagonalization gives

$$
P_{R}(y)=T(y, f(y))=\neg T(y, f(y))
$$

The test function $T(y, f(y))$ is undecidable. The rule for deciding if a number is Richardian is the negation of itself. However, the key point is not that $T(y, f(y))$ is undecidable, but that it is semantically meaningless and the undecidability is merely a consequence of that or an indication of that. When faced with some natural number and its map statement "A Richardian number is one not described by the statement it maps to" there is no logically prior information to decide if the number is Richardian. It is a meaningless statement in that context. A map is not logically prior to its contents. Just as in the proof of Cantor's theorem there is no information to decide if $y \in f(y)$ because $y$ lies outside the map. The logical order is contents $\rightarrow$ map $\rightarrow$ outside. To ignore this fact and start to play logic games by supposing that it were true, only to discover that it must then be false, is to entirely miss the point. Semantic diagonalization always involves using a rule to determine the meaning of an object by referring it to itself and so is not valid purely on semantic grounds. The contradiction, as with all semantic diagonalizations, is a result of failing to understand the logical ordering of the problem set-up. It doesn't actually exist and so nothing can be meaningfully concluded from it, other than something about the problem set-up has not been properly understood. Richard considered that the resolution is that the problematic map item is not just a property of the numbers within the map, but also a property of the map itself. This is because the ordering is arbitrary. As such, the placement of the Richardian statement is not arbitrary. It can only meaningfully refer to an object that exists. So the statement can only be added after the map is completed which will never happen. In both the proof of Cantor's theorem and the Richardian number paradox, the ordering of the list is entirely arbitrary. Thus, $y$ could potentially be anywhere inside the map and so must be nowhere.

Gödel's incompleteness theorem [Gödel,1931] considers a map from the natural numbers to any valid expression in some formal language, $L$. However, in this case, the natural number assignment to an expression is not arbitrary. The proof considers that there is a method for encoding a unique number, called its Gödel number, for any given expression and decoding it back to the expression. Not only can expressions be encoded, but also sequences of expressions that constitute a proof. The system is also set up in such a way that there is an isomorphism $(I)$ between the Gödel number space and the expression space such that all operations can be carried out merely by manipulating or comparing numbers in the Gödel number space. The Gödel numbers for expressions and sequences of expressions are constructed by concatenating together, in a reversible fashion, the numbers assigned to the defined primitive symbols of the language, which include symbols for representing free variables. Formulas as functions of free variables can also be evaluated purely within the Gödel number space. For example, if $f(x)$ is some expression of a free variable $x$ and [] returns the Gödel number of any expression, then the expression can be evaluated for some concrete number $m$ as $[f(x)] \cdot R_{G}([x],[m])=[f(m)]$ where $n \cdot R_{G}(a, b)$ means replace, in the $G$ space, each found value of $a$, inside $n$, with the value of $b$. This corresponds with the $L$ space operation of replacing the " $x$ " symbol with the "m" symbol. Some key properties follow from this: 
- Only the exact same sequence of language symbols can be encoded by the same Gödel number. Any change of symbol in the $L$ space results in a corresponding change of number in the $G$ space. This is essential for reversibility since otherwise there would be no unique way to decode a given Gödel number.

- Reversibility implies that all maps are bi-directional. Since all statements are constructed in a well-founded manner all maps must be well-founded. There can be no infinite descending chains of maps back to the starting language symbols. Consequently no Gödel number can map to a statement that contains the Gödel number of the statement. No amount of reversible construction steps can produce an irreversible result.

The system is flexible enough to contain expressions about Gödel numbers and those expressions will have their own Gödel number and so on. To see it in operation, the proof proceeds as follows:

$\begin{array}{lll}\text { Gödel number space } & & \text { L space } \\ x & \rightarrow & \text { an expression in the language } \\ y & \rightarrow & \text { a proof of an expression } \\ p & \rightarrow & \operatorname{Proof}(x, y) \\ q & \rightarrow & \neg \operatorname{Provable}(x)\end{array}$

The expression Proof $(x, y)$ is actually a test of an arithmetic relationship between $x$ and $y$ and it is assumed here that it can be shown that it will only be true when $y$ is a proof of $x$. Provable $(x)$ is an arithmetic test on $x$ that will be true when $x$ has a proof. That is, there is some $y$ that is a proof of $x, \exists y: \operatorname{Proof}(x, y)$. The last expression is the statement that some expression in the language is not provable, whenever the last expression evaluates to true. That is, $\neg$ Provable $(x)=\forall y \neg \operatorname{Proof}(x, y)$.

Gödel systems do not typically evaluate true and false propositions. This is because there is no single Gödel number for true or false as can be seen from the isomorphism. What they do is circumvent the issue by making assertions. Assertions are not true or false. They are either correct or incorrect, although the reason they are called assertions is that one is typically expected to consider them correct. Note that all proofs are assertions. An assertion is a statement that a proposition is true. Only it or its negation can be correct. This means one can treat assertions as logical propositions, since a correct assertion is semantically equivalent to a proposition being true and an incorrect assertion is semantically equivalent to a proposition being false. Practically, the distinction means that it is assumed there are no functions within $L$ that evaluate assertions and all provable assertions are indeed considered to be correct. That is, consistent with the axioms from which they were built, since the act of proving any statement involves tracking it back to axioms via a sequence of relatable statements. This is precisely what is encoded into the $p$ statement. Statements over free variables are different. They are predicates, with a potential mix of true and false propositions ranging over each value of a free variable. To convert to an assertion, the assertion is on any value of the free variable. For example the assertion Provable $(x)$ is not the assertion that some $x$ is provable. It is the assertion that any $x$ is provable and will clearly be an incorrect assertion for many values of $x$. For such an assertion to be provable it must be provable for all values of the free variable. To see how assertions over a free variable 
work, consider the following statements

$\begin{array}{lll}\text { G space } & & \text { L space } \\ a & \rightarrow & x>2 \\ n a & \rightarrow & x \leq 2\end{array}$

Note that since assertions apply to any $x$, the na assertion is not strictly the negation of the $a$ assertion. The negation is the negation of the L space statement. Assertions are only equivalent to their L space statements when there are no free variables. In order for the $a$ or $n a$ statements to be provable they must be provable for all values of the free variable. There are 4 possibilities

$$
\begin{aligned}
& \operatorname{Provable}(a)=\text { true }=\text { all } x>2 \\
& \operatorname{Provable}(a)=\text { false }=\text { some } x<=2 \\
& \operatorname{Provable}(n a)=\text { true }=\text { all } x<=2 \\
& \operatorname{Provable}(n a)=\text { false }=\text { some } x>2
\end{aligned}
$$

Note that whenever a statement is provable then its L space negation is not provable. But there does exist a consistent solution in which both statements are not provable. This is just a consequence of the two statements not describing opposing assertions. The $q$ statement in Equation 6.5 is a statement that ranges over a free variable. For the $q$ statement the assertion is

$$
\begin{array}{ll}
\text { G space } & \text { L space } \\
q & \rightarrow \quad \neg \operatorname{Provable}(x)
\end{array}
$$

The provability of the assertion is given by

$$
\begin{aligned}
& \operatorname{Provable}(q)=\text { true }=\text { all } x \text { not provable } \\
& \operatorname{Provable}(q)=\text { false }=\text { some } x \text { provable }
\end{aligned}
$$

and so

$$
\begin{aligned}
& \neg \operatorname{Provable}(q)=\text { true }=\text { some } x \text { provable } \\
& \neg \operatorname{Provable}(q)=\text { false }=\text { all } x \text { not provable }
\end{aligned}
$$

Asserting that $q$ is not provable is equivalent to asserting that some $x$ are provable, which is generally the case for statements over free variables. Consequently, the assertion that $q$ is not provable is a correct assertion and provably so.

The Gödel sentence for L, labelled here as $G_{s}$, is the $q$ statement evaluated for itself.

$$
G_{s}=\neg \operatorname{Provable}(q)
$$

The $G_{s}$ assertion is " $q$ is not provable". There is no self reference in this version. It is not possible to get a direct self reference in a Gödel system because the Gödel number within a statement is never equal to the Gödel number of the statement since that would be a violation of the isomorphism. No sequence of symbols can ever contain itself. This 
prohibits any possibility of a self reference except for those statements that are correlated with other statements that reference them. For example, it is not possible to construct the assertion "I am false" even if there were to be made available true() and false() functions of Gödel numbers within $L$. A self reference requires a statement to be correlated with another statement that references it. Consider the statements

$\begin{array}{lll}\text { G space } & & \text { L space } \\ q & \rightarrow & \neg \operatorname{Provable}(x) \\ {\left[G_{s}^{1}\right]} & \rightarrow & \neg \operatorname{Provable}(q) \\ {\left[G_{s}^{2}\right]} & \rightarrow & \neg \operatorname{Provable}\left(\left[G_{s}^{1}\right]\right)\end{array}$

Note that $q \neq\left[G_{s}^{1}\right] \neq\left[G_{s}^{2}\right]$. This should be obvious from the isomorphism, since the Gödel number for each statement is obtained by taking $q$ and replacing all instances of the Gödel number for $x$ along $q$ with the Gödel number of the argument. Specifically, $\left[G_{s}^{1}\right]$ cannot be equal to $q$, since that would require the symbol for a free variable to be the same as the symbol for some particular number. $G_{s}^{1}$ is true and provable and so $G_{s}^{2}$ must be false. Thus $G_{s}^{1}=\neg G_{s}^{2}$ and so $G_{s}^{1}=\operatorname{Provable}\left(\left[G_{s}^{1}\right]\right)$. The negation of the $G_{s}^{2}$ assertion, which is equal to the $G_{s}^{1}$ assertion, is " $G_{s}^{1}$ has a proof". Thus, for $G_{s}=G_{s}^{1}$

$$
\begin{aligned}
G_{s} & =\operatorname{Provable}\left(\left[G_{s}\right]\right) \\
& =\text { "I am provable" }=" q \text { is not provable" }=\text { "some } x \text { 's are provable" }
\end{aligned}
$$

So $G_{s}$ is true and provable.

The proof of the incompleteness theorem attempts to engineer the negation of a self reference and claims to have constructed the opposite statement to that above. This must not be possible since the two statements could then be combined to obtain $G_{s}=\neg G_{s}$. The opposing statement is given by

$$
G_{s}^{\star}=\neg \operatorname{Provable}\left(\left[G_{s}^{\star}\right]\right)
$$

where the $\star$ is merely to indicate a non-constructable statement. It has some peculiarities of its own. It is a contradiction of standard logic since the assertion is incorrect when provable and correct when not provable. Also, when the assertion "I am not provable" is true, then I is not provable. But when I also refers to the statement itself, then its negation must be provable and so I must also be provable. Thus, it is a logical impossibility for the "I" in "I am not provable" to refer to the statement of which it is a member. The only possible escape from this conclusion is if there exist assertions for which neither the assertion nor its negation can be proved and the assertion "I am not provable" is a specific example. However, that is not possible in this case since it has already been shown that the assertion "I am provable" is provable. Whether or not any such assertions can exist is considered later. To compound the issue, the original proof additionally relies on the map

$$
\begin{array}{ll}
\text { G space } & \text { L space } \\
{\left[G_{s}^{\star}\right] \quad \rightarrow \quad \neg \operatorname{Provable}\left(\left[G_{s}^{\star}\right]\right)}
\end{array}
$$

This is a violation of the isomorphism since it involves a map that is not well-founded. 
The contained sequence of symbols in $\left[G_{s}^{\star}\right]$ endlessly repeats and so $\left[G_{s}^{\star}\right]$ is not reversible to a starting sequence of symbols. It can only be arrived at from $G_{s}^{2}=\neg \operatorname{Provable}\left(\left[G_{s}^{1}\right]\right)$ by equivocating on $G_{s}^{2}=G_{s}^{1}$, which is false, and $\left[G_{s}^{2}\right]=\left[G_{s}^{1}\right]$, which is also false. Even if Equation 6.12 were possible, it would still mean that the assertion was incorrect when provable. The proof considers that if $G_{s}^{\star}$ is provable then its assertion is considered correct which is $\forall y \neg \operatorname{Proof}\left(\left[G_{s}^{\star}\right], y\right)$, but if it is provable then there should be some $n$ that proves it: $\exists n: \operatorname{Proof}\left(\left[G_{s}^{\star}\right], n\right)$. The two assertions are inconsistent. The proof then concludes that $G_{s}^{\star}$ cannot be provable and since its self reference version says that it is not provable, it is a correct statement. However, that is not a valid inference. The truth of a statement cannot be inferred from its non-provability except for a complete axiomatic system and such a system expressly forbids true statements that are not provable. Of course, it has been shown here that $G_{s}$ is true by a different process. Thus, it is concluded that the language contains a true statement that is not provable. The solution to this in the standard approach is to say that the statement is only not provable within the defined language. That is, there are a hierarchy of languages. A so called "meta-language" is required to inject additional axioms which enable the statement to be proved. But then one can form a Gödel sentence of this language and so a meta-meta-language is now required and so on. This is equivalent to the relentless re-appearance of the Burali-Forti paradox with each new class type, in the standard approach.

The standard proof creates a semantic diagonalization where there should be none by simultaneously assuming $G_{s}=\operatorname{Provable}\left(\left[G_{s}\right]\right)$ and $G_{s}=\neg \operatorname{Provable}\left(\left[G_{s}\right]\right)$, which results in $G_{s}=\neg G_{s}$. It creates a logic trap for itself by assuming that a provable statement must also be a correct assertion and then makes use of an assertion that is not only invalid but also violates the initial assumption.

In Gödel's original proof [Gödel,1931], adding $Z()$ as well as [] for Gödel numbers of statements, then Gödel's relation sign $q=[\neg \operatorname{Proof}(y, x)]$, class sign $p=[\neg \operatorname{Provable}(y)]$, recursive class sign $r=[\neg \operatorname{Proof}(p, x)]$ and $17 G e n r=[\neg$ Provable $(p)]$. Note that $x, y$ and $p, q$ have switched roles from earlier with $q$ also acquiring a negation. Equation (9) is the statement that $\neg \operatorname{Proof}\left(S b\left(y_{Z(y)}^{19}\right), x\right) \Rightarrow \operatorname{Provable}\left(S b\left(q_{Z(x)}^{17} \underset{Z(y)}{19}\right)\right)$, with $S b\left(n_{b}^{a}\right)=n . R_{G}(a, b)$ and 17 is the Gödel number of the language symbol " $x$ " and 19 is the Gödel number of the language symbol "y". Note that whenever $Z(x)$ or $Z(y)$ appears, it is implied to be $Z$ ("x") or $Z$ ("y"). Thus, Equation (9) simplifies to $\neg \operatorname{Proof}(y, x) \Rightarrow \operatorname{Provable}([\neg \operatorname{Proof}(y, x)])$, which is that when $x$ is not a proof of $y$ then there is a proof of $x$ not being a proof of $y$. Equation (15) should then have been the same statement with $y$ replaced by $p$, but $p=[\neg$ Provable $(p)]$ is introduced by saying that in Equation (9) that $y$ is replaced with $p$, but actually proceeding on the basis that on the left hand side it is replaced with $[\neg$ Provable $(p)]$. That is, the implied though never written and incorrect transformation of the substitution $S b\left(y_{Z(y)}^{19}\right) \rightarrow S b\left(p_{Z(p)}^{19}\right)$ results in $y$ being replaced with $p$ and then the $y$ inside $p$ is replaced with $p$ and so ceases to be $p$ since any change of symbol produces a different Gödel number. The transformation of the substitution should have been $S b\left(y_{Z(\text { "y" })}^{19}\right) \rightarrow S b\left(p_{Z(\text { "y" })}^{19}\right)$. It simply cannot be that $p$ which is defined to be $[\neg$ Provable $(y)]$ is then also equal to $[\neg$ Provable $(p)]$, since that would require the symbol for a free variable to be the same as the symbol for some particular number. Gödel numbers are required, by design, to decode to a unique sequence of language symbols. 
The relationship between an assertion, $A$, and its provability can be explored by constructing a table of all possible true/false permutations for $A$, Provable $(A), \neg A, \operatorname{Provable}(\neg A)$. The table will have 8 rows. 4 of the rows can be eliminated by imposing the constraint that a false assertion cannot be proved, that is $\neg A=>\neg \operatorname{Provable}(A)$ or the constraint that if an assertion is provable then it must be axiomatically correct since the act of proving any statement involves showing a path exists from the axioms to the statement and so Provable $(A)=>A$. The remaining rows describe two possible cases. The first case is that the provability of an assertion is equivalent to its correctness

$$
\operatorname{Provable}(A)=A
$$

The second case is that neither the assertion nor its negation are provable

$$
\operatorname{Provable}(A)=\operatorname{Provable}(\neg A)=\text { false }
$$

The absence of Equation 6.14 is equivalent to the definition of completeness for an axiomatic system. Its existence can be trivially engineered by taking a complete axiomatic system and deleting one of the axioms from the system. The system is then trivially incomplete. The incompleteness theorem explores whether there are any non-trivial examples. Whether there are systems that can never be made complete no matter how many axioms are added. However, it fails to prove its case. The statement that the incompleteness theorem claims to have constructed is a statement that cannot actually be constructed. If every system can be made complete by a suitable choice of axioms then "provable" and "correct" are synonyms of each other. In a fully deductive system, both truth and provability expand out together from the axioms. If $d$ is the Gödel number of a primitive definition, then it can be arranged so that all primitive definitions are their own proof by setting $\operatorname{Proof}(d, d)=$ true. That is, one is free to consider that a statement has a proof if it is either an axiom or tracks back to only axioms via a sequence of relatable statements. Gödel's original system did this. In practice, using assertions just means amending the $p$ assertion to include a test for $d$. Now all provable statements have a provable anchor just as all true statements have a true anchor and it is the same anchor. In a deductive system all truths expand out from the axioms and every truth is that which is deduced from logically prior truths and hence proved. That is not to say that any statement can be easily proved or disproved. Finding a path backwards through unknown territory can be just as difficult as mapping out the territory by generating all the possible truths that follow from a set of axioms. But if the system is fully deductive and hence complete then Equation 6.14 is evidence of an error just as much as $A=\neg A$.

A semantic diagonalization should be suspected any time an object is claimed to be the opposite of itself. It simply cannot happen. Another example is the halting problem. Consider a computer program halt $([P],[I])$ that takes the source code $[P]$ of some program $P$, as input, and evaluates whether or not, when $P$ runs, if it halts when acting on some input $[I]$. To make it a logic formulation, consider that all other functions are proxy halt functions that return true if they halt and false otherwise. A function can be constructed that returns the opposite of whether some $[P]$ halts when run on its own source code as input:

$$
\operatorname{neg} H([P])\{\operatorname{return} \neg \operatorname{halt}([P],[P])\}
$$


The halting problem is that halt $([n e g H],[n e g H])$ returns the opposite of itself and so such a halt function is logically impossible. However, all it really does is return some value based on some text input and all negH([negH]) does when it runs is return the opposite of whatever the halt function says it does. Nothing will be acting as the negation of itself. Neither is there any logical contradiction. The halt function is merely an assertion and within negH([negH]) it is clearly an incorrect assertion. The halt inside negH() is logically distinct from a halt acting outside $n e g H()$. A fact which is made explicit by adding an argument for the identifier of the calling routine. For then the relationship is:

$$
\operatorname{halt}(@ \operatorname{main},[n e g H],[n e g H]=\neg \text { halt }(@ n e g H,[n e g H],[n e g H])
$$

A semantic diagonalization is to assume that both functions are the same. The distinction makes it possible construct a halt function that correctly predicts the output of negH([negH]) by returning a different value depending on its location, although a practical application would more likely rely on @main to quarantine bad code.

In the original version of the halting problem [Turing,1937], Turing first considers a computing device capable of printing out all computable numbers as sequences of 1's and 0's and further considers a map from the natural numbers to the sequences. $\alpha_{n}$ denotes the $\mathrm{n}^{\text {th }}$ sequence in the list and $\phi_{n}(m)$ denotes the $\mathrm{m}^{\text {th }}$ digit of the $\alpha_{n}$ sequence or the last digit if there are fewer digits else 0 . If every sequence is computable then there is some other sequence, $\beta$, with its $\phi(n)$ digit that is different from that of $\alpha_{n}$. That is, $\beta(n)=1-\phi_{n}(n)$. If $\beta$ is computable, then it is equal to some $\alpha_{k}$ and so $\phi_{k}(n)=1-\phi_{n}(n)$. Turing writes that this is true for all $n$ and then sets $n$ to $k$ to give $2 \phi_{k}(k)=1$, which is not possible and so concludes that $\beta$ is not computable. The $\beta$ sequence digits can be treated as boolean values to obtain $\beta(n)=\neg \phi_{n}(n)$ and since $\beta=\alpha_{k}$ then $\phi_{k}(n)=\neg \phi_{n}(n)$. This is an expression that ranges over a free variable $n$. Setting $n$ to $k$ gives $\phi_{k}(k)=\neg \phi_{k}(k)$. Here is another semantic diagonalization. However, since $\beta=\alpha_{k}$ and $\beta$ is some other sequence different from $\alpha_{n}$ then it is clear that the one thing that is forbidden is setting $n$ to $k$. The rule for $\beta$, that is, $\beta(n)=\neg \phi_{n}(n)$, is identical to the diagonal rule for computing the $T$ set of a matrix of 1 's and 0's. Thus, $\beta$ always tracks below the diagonal of the map and there is no contradiction.

The halting argument is somewhat different and instead constructs a different sequence $\beta^{\prime}$. Consider a halt program $H_{M}$ that analyses some code $M$, decides if it halts and if it does then it writes out one of the digits of the output from $M$ such that $\beta^{\prime}(r)=\phi_{r}(r)$, where $r$ is the index count of each $M$ that halts. A code is considered to halt even if it requires an infinite amount of time to complete provided it does not get trapped in a repeating loop. The instructions for any computation are supplied on tape to a finite state automata machine and are shown here as being supplied left first. The details are not important. It is assumed the instructions work as described. The instructions for $H_{M}$ are $H_{M}=O M U M$, where $M$ is the code to analyse and $O$ are the instructions to decide if $M$ halts. If it decides that $M$ doesn't halt then $O$ halts. If it decides that $M$ halts then the computation proceeds with the $U M$ instructions that write out the $\mathrm{r}^{\text {th }}$ digit of the output of $M$ or the last digit if $M$ completes with fewer digits or else 0 if it completes with no output. Thus, $H_{M}$ always halts. Turing conceived of a grand $H$ for computing all of $\beta^{\prime}$ in one go as $H=O M_{1} U M_{1} O M_{2} U M_{2} \ldots$, where if $O$ halts it first moves the tape to the next $H_{M}$ and the $M_{i}$ entries cover all coding 
sequences containable within $H$. The halting argument requires assuming that some $M_{i}$ within $H$ is equal to $H$. However, this is impossible. In the construction of $H$, only objects that already exist can be added and every addition creates a new $\mathrm{H}$ different from every added object. Coding instructions make for ideal components in Gödel numbers and the Gödel number within a statement is never the same as the Gödel number of a statement. No sequence of language symbols can ever contain itself. $H$ can never contain itself just as no set can contain itself. Nothing can ever contain itself as that would be a violation of the law of identity. Two things cannot be the same thing if they have different properties and location/neighbourhood and hierarchy/rank are properties. In any descriptive language that can be formalized, the container of things will have a different Gödel number to the things it contains. Both the halting argument and Gödel's incompleteness theorem require a sequence of language symbols to contain itself, which is logically impossible. Thus, $[H]$ will never contain itself. If it did and say it was $K$, then $K$ would be an impossible sequence that endlessly contains itself and $H$ would be doomed to endlessly repeat itself each time it reached itself somewhere within its list of instructions and so could never compute the $r(K)^{t h}$ digit of $\beta^{\prime}$. $O$ cannot simply skip over the contained $H$ since then the grand $H$ will halt. But this will never happen. Thus, $H$ will have no difficulty printing out all of $\beta^{\prime}$ since, by definition, every single $O M U M$ either prints out a digit or moves to the next $O M U M$. The $\beta^{\prime}$ sequence is really irrelevant to the argument, which is about whether or not the $H$ sequence will consistently halt. $U$ could simply run $M$ to completion.

Consequently, a halt function is possible for a similar reason that all axiomatically correct statements are provable. In an axiomatic description of a computing device in which all correct statements are provable then either the assertion halt $([P],[I])$ or its negation, for some $[P]$ and $[I]$, will be provable and hence computable. 


\section{$7 \quad$ References}

1. G. Cantor: Über eine Eigenschaft des Inbegriffes aller reellen algebraischen Zahlen, Journal für die Reine und Angewandte Mathematik, Vol77 (1874) pp. 258-262

2. G Cantor: Über eine elementare Frage der Mannigfaltigkeitslehre, Jahresbericht der Deutschen Mathematiker-Vereinigung, Vol 1 (1891) pp. 75-78

3. G. Hessenberg: Grundbegriffe der Mengenlehre, Abhandlungen der Friesschen Schule, Neue Folge 1, Heft IV (1906) pp. 478-706

4. A. Weir: Naive Set Theory is Innocent!, Mind, Vol. 107.428 (1998) pp. 763-798

5. J. Richard: Les Principes des Mathématiques et le Problème des Ensembles, Revue Générale des Sciences Pures et Appliquées, vol 16 (1905) pp. 541

6. K. Gödel: Über formal unentscheidbare Sätze der Principia Mathematica und verwandter Systeme, I, Monatshefte für Mathematik und Physik, vol 38 (1931) pp. 173-198

7. A. Turing: On Computable Numbers with an Application to the Entscheidungsproblem, Proceedings of the London Mathematical Society, Ser. 2, Vol. 42 (1937) pp. 230-265 